\title{
A dynamic unilateral contact problem with adhesion and friction in viscoelasticity
}

\author{
Marius Cocou. 1 Mathieu Schryve? and Michel Raous 3
}

Mathematics Subject Classification (2000). 35K85, 35R35, 49J40, 74A55, 74D05, 74H20

Keywords. Unilateral contact, adhesion, healing, friction, dynamic problems, viscoelasticity.

\begin{abstract}
The aim of this paper is to study an interaction law coupling recoverable adhesion, friction and unilateral contact between two viscoelastic bodies of Kelvin-Voigt type. A dynamic contact problem with adhesion and nonlocal friction is considered and its variational formulation is written as the coupling between an implicit variational inequality and a parabolic variational inequality describing the evolution of the intensity of adhesion. The existence and approximation of variational solutions are analysed, based on a penalty method, some abstract results and compactness properties. Finally, some numerical examples are presented.
\end{abstract}

\section{Introduction}

In this paper we consider an interaction law including dynamic unilateral contact, recoverable adhesion and nonlocal friction between two viscoelastic bodies. The adhesion is characterized by the intensity of adhesion, first introduced by M. Frémond (see, e.g., [13, 14]). An interface law for a quasistatic evolution where rebonding is not allowed was originally proposed in [22] in the framework of continuum thermodynamics. The corresponding quasistatic problem coupling unilateral contact, adhesion and local friction

\footnotetext{
${ }^{1}$ Laboratoire de Mécanique et d'Acoustique CNRS, 31 chemin Joseph Aiguier, 13402 Marseille Cedex 20 and Université de Provence, UFR - MIM, Marseille, France

${ }^{2}$ Laboratoire de Mécanique et d'Acoustique CNRS, 31 chemin Joseph Aiguier, 13402 Marseille Cedex 20 and Université de Provence, UFR - MIM, Marseille, France

${ }^{3}$ Laboratoire de Mécanique et d'Acoustique CNRS, 31 chemin Joseph Aiguier, 13402 Marseille Cedex 20, France E-mails: cocou, schryve, raous@lma.cnrs-mrs.fr
} 
for an elastic body has been analysed in 8. This interface law has been used on various applications : fibre-matrix interaction in the context of composite materials [24, steel-concrete interaction [23] and, more recently, tectonic plates interaction in the context of earthquakes.

The extension to reversible adhesion proposed in the present paper constitutes an approximation of interactions of van der Waals type for rubber glass contact and other specific phenomena as those studied by M. Barquins [3] and K.L. Johnson, K. Kendall, A.D. Roberts [16, 17]. This model, which extends the one considered in [22, 24], is dedicated to the description of bond damage and can be called a healing model because it allows us to describe both the formation and the rupture of adhesive contacts during the approach of the bodies. Also, in this model bond damage and healing behaviours are rate-dependent. The dissipation effects are related to both viscoelasticity and adhesion.

Dynamic frictional contact problems with normal compliance laws for a viscoelastic body have been considered by J.A.C. Martins and J.T. Oden [20], K.L. Kuttler [18], O. Chau, W. Han and M. Sofonea [6]. Dynamic unilateral or bilateral contact problems with friction for viscoelastic bodies have been studied in [12, 19, 9] and dynamic frictionless problems with adhesion have been analysed in [28] and in the references therein.

In this work we consider a coupled dynamic problem combining reversible adhesion, friction and unilateral contact. In section 2, classical and variational formulations of the dynamic contact problem are presented. The variational formulation is given as an implicit variational inequality coupled with a parabolic variational inequality which describes the evolution of the intensity of adhesion. Also, classical and variational formulations of an auxiliary penalized problem are considered. In section 3, general existence and uniqueness results are proved. In section 4 , these abstract results are used to prove the existence and the uniqueness of penalized solutions and the existence of solutions to unilateral contact problem. In section 5 , some numerical examples are presented and discussed.

\section{Classical and variational formulations}

We consider two viscoelastic bodies, characterized by a Kelvin-Voigt constitutive law, which occupy the reference domains $\Omega^{\alpha}$ of $\mathbb{R}^{d}, d=2$ or 3 , with Lipschitz continuous boundaries $\Gamma^{\alpha}=\partial \Omega^{\alpha}, \alpha=1,2$. In this paper we assume the small deformation hypothesis and we use Cartesian coordinate representations.

Let $\Gamma_{U}^{\alpha}, \Gamma_{F}^{\alpha}$ and $\Gamma_{C}^{\alpha}$ be three open disjoint sufficiently smooth parts of $\Gamma^{\alpha}$ 
such that $\Gamma^{\alpha}=\bar{\Gamma}_{U}^{\alpha} \cup \bar{\Gamma}_{F}^{\alpha} \cup \bar{\Gamma}_{C}^{\alpha}$ and, to simplify the estimates, meas $\left(\Gamma_{U}^{\alpha}\right)>$ $0, \alpha=1,2$.

We denote by $\boldsymbol{y}^{\alpha}\left(\boldsymbol{x}^{\alpha}, t\right)$ the position at time $t \in[0, T]$, where $T>0$, of the material point represented by $\boldsymbol{x}^{\alpha}$ in the reference configuration, by $\boldsymbol{u}^{\alpha}\left(\boldsymbol{x}^{\alpha}, t\right):=\boldsymbol{y}^{\alpha}\left(\boldsymbol{x}^{\alpha}, t\right)-\boldsymbol{x}^{\alpha}$ the displacement vector of $\boldsymbol{x}^{\alpha}$ at time $t$, with the Cartesian coordinates $u^{\alpha}=\left(u_{1}^{\alpha}, \ldots, u_{d}^{\alpha}\right)=\left(\bar{u}^{\alpha}, u_{d}^{\alpha}\right)$. Let $\varepsilon^{\alpha}$, with the Cartesian coordinates $\varepsilon^{\alpha}=\left(\varepsilon_{i j}\left(u^{\alpha}\right)\right)$, and $\boldsymbol{\sigma}^{\alpha}$, with the Cartesian coordinates $\sigma^{\alpha}=\left(\sigma_{i j}^{\alpha}\right)$, be the infinitesimal strain tensor and the stress tensor, respectively, corresponding to $\Omega^{\alpha}, \alpha=1,2$.

Excepting in the last section, to simplify notations we assume that the displacement $\boldsymbol{U}^{\alpha}=\mathbf{0}$ is prescribed on $\left.\Gamma_{U}^{\alpha} \times\right] 0, T[, \alpha=1,2$, and that the densities of both bodies are equal to 1. Let $\boldsymbol{f}=\left(\boldsymbol{f}^{1}, \boldsymbol{f}^{2}\right)$ and $\boldsymbol{F}=\left(\boldsymbol{F}^{1}, \boldsymbol{F}^{2}\right)$ denote the given body forces in $\Omega^{1} \cup \Omega^{2}$ and tractions on $\Gamma_{F}^{1} \cup \Gamma_{F}^{2}$, respectively. The initial displacements and velocities of the bodies are denoted by $\boldsymbol{u}_{0}=$ $\left(\boldsymbol{u}_{0}^{1}, \boldsymbol{u}_{0}^{2}\right), \boldsymbol{u}_{1}=\left(\boldsymbol{u}_{1}^{1}, \boldsymbol{u}_{1}^{2}\right)$. The usual summation convention will be used for $i, j, k, l=1, \ldots, d$.

We suppose that the solids can be in unilateral contact between the potential contact surfaces $\Gamma_{C}^{1}$ and $\Gamma_{C}^{2}$. We assume also that the surfaces $\Gamma_{C}^{1}$ and $\Gamma_{C}^{2}$ can be parametrized by two $C^{1}$ functions, $\varphi^{1}, \varphi^{2}$, defined on an open subset $\Xi$ of $\mathbb{R}^{d-1}$ such that $\varphi^{1}(\xi)-\varphi^{2}(\xi) \geq 0 \forall \xi \in \Xi$ and each $\Gamma_{C}^{\alpha}$ is the graph of $\varphi^{\alpha}$ on $\Xi$ that is $\Gamma_{C}^{\alpha}=\left\{\left(\xi, \varphi^{\alpha}(\xi)\right) \in \mathbb{R}^{d} ; \xi \in \Xi\right\}, \alpha=1,2$. Let $\boldsymbol{m}^{\alpha}: \Xi \rightarrow \mathbb{R}^{d}$, with $\boldsymbol{m}^{1}(\xi):=\left(\nabla \varphi^{1}(\xi),-1\right), \boldsymbol{m}^{2}(\xi):=\left(-\nabla \varphi^{2}(\xi), 1\right)$, $\forall \xi \in \Xi$, be the outward normal to $\Gamma_{C}^{\alpha}, \alpha=1,2$. Since the displacements, their derivatives and the gap are assumed small, by using a similar method as the one considered by P. Boieri, F. Gastaldi and D. Kinderlehrer [4] (see also [9]) we obtain the following contact condition at time $t$ on the set $\Xi$ :

$$
\begin{aligned}
0 & \leq \varphi^{1}(\xi)-\varphi^{2}(\xi)+u_{d}^{1}\left(\xi, \varphi^{\alpha}(\xi), t\right)-u_{d}^{2}\left(\xi, \varphi^{\alpha}(\xi), t\right) \\
& -\nabla \varphi^{1}(\xi) \cdot \bar{u}^{1}\left(\xi, \varphi^{1}(\xi), t\right)+\nabla \varphi^{2}(\xi) \cdot \bar{u}^{2}\left(\xi, \varphi^{2}(\xi), t\right) \quad \forall \xi \in \Xi,
\end{aligned}
$$

or, using the definition of $\boldsymbol{m}^{1}, \boldsymbol{m}^{2}$,

$\boldsymbol{m}^{1}(\xi) \cdot \boldsymbol{u}^{1}\left(\xi, \varphi^{1}(\xi), t\right)+\boldsymbol{m}^{2}(\xi) \cdot \boldsymbol{u}^{2}\left(\xi, \varphi^{2}(\xi), t\right) \leq \varphi^{1}(\xi)-\varphi^{2}(\xi) \quad \forall \xi \in \Xi(2.1)$

Let $\boldsymbol{n}^{\alpha}:=\boldsymbol{m}^{\alpha} /\left|\boldsymbol{m}^{\alpha}\right|$ denote the unit outward normal vector to $\Gamma_{C}^{\alpha}, \alpha=1,2$. The initial normalized gap between the two contact surfaces is defined as

$$
g_{0}(\xi):=\frac{\varphi^{1}(\xi)-\varphi^{2}(\xi)}{\sqrt{1+\left|\nabla \varphi^{1}(\xi)\right|^{2}}} \quad \forall \xi \in \Xi .
$$

We shall use the following notations for the normal and tangential components of a displacement field $\boldsymbol{v}^{\alpha}, \alpha=1,2$, of the relative displacement 
corresponding to $\boldsymbol{v}:=\left(\boldsymbol{v}^{1}, \boldsymbol{v}^{2}\right)$, by including the initial gap $g_{0}$ in the normal direction, and of the stress vector $\boldsymbol{\sigma}^{\alpha} \boldsymbol{n}^{\alpha}$ on $\Gamma_{C}^{\alpha}$ :

$$
\begin{aligned}
& \boldsymbol{v}^{\alpha}:=\boldsymbol{v}^{\alpha}(\xi, t)=\boldsymbol{v}^{\alpha}\left(\xi, \varphi^{\alpha}(\xi), t\right), \\
& v_{N}^{\alpha}:=v_{N}^{\alpha}(\xi, t)=\boldsymbol{v}^{\alpha}\left(\xi, \varphi^{\alpha}(\xi), t\right) \cdot \boldsymbol{n}^{\alpha}(\xi), \quad \boldsymbol{v}_{T}^{\alpha}:=\boldsymbol{v}_{T}^{\alpha}(\xi, t)=\boldsymbol{v}^{\alpha}-v_{N}^{\alpha} \boldsymbol{n}^{\alpha}, \\
& {\left[v_{N}\right]:=\left[v_{N}\right](\xi, t)=v_{N}^{1}+v_{N}^{2}-g_{0}, \quad\left[\boldsymbol{v}_{T}\right]:=\left[\boldsymbol{v}_{T}\right](\xi, t)=\boldsymbol{v}_{T}^{1}-\boldsymbol{v}_{T}^{2},} \\
& \sigma_{N}^{\alpha}:=\sigma_{N}^{\alpha}(\xi, t)=\left(\boldsymbol{\sigma}^{\alpha} \boldsymbol{n}^{\alpha}\right) \cdot \boldsymbol{n}^{\alpha}, \quad \boldsymbol{\sigma}_{T}^{\alpha}:=\boldsymbol{\sigma}_{T}^{\alpha}(\xi, t)=\boldsymbol{\sigma}^{\alpha} \boldsymbol{n}^{\alpha}-\sigma_{N}^{\alpha} \boldsymbol{n}^{\alpha},
\end{aligned}
$$

for all $\xi \in \Xi$ and for all $t \in[0, T]$. We denote also by $g:=-\left[u_{N}\right]=$ $g_{0}-u_{N}^{1}-u_{N}^{2}$ the gap corresponding to the solution $\boldsymbol{u}:=\left(\boldsymbol{u}^{1}, \boldsymbol{u}^{2}\right)$. Assuming that $\nabla \varphi^{1}(\xi) \simeq \nabla \varphi^{2}(\xi)$, it follows that the unilateral contact condition (2.1) at time $t$ can be written as

$$
\left[u_{N}\right](\xi, t)=-g(\xi, t) \leq 0 \quad \forall \xi \in \Xi
$$

Following M. Frémond [13, 14], we introduce the internal state variable $\beta$, which represents the intensity of adhesion $(\beta=1$ means that the adhesion is total, $\beta=0$ means that there is no adhesion and $0<\beta<1$ is the case of partial adhesion). In the following, we will consider only isothermal evolutions.

\subsection{Classical formulation}

Let $\mathcal{A}^{\alpha}, \mathcal{B}^{\alpha}$ denote two fourth-order tensors, the elasticity tensor and the viscosity tensor corresponding to $\Omega^{\alpha}$, with the components $\mathcal{A}^{\alpha}=\left(\mathcal{A}_{i j k l}^{\alpha}\right)$ and $\mathcal{B}^{\alpha}=\left(\mathcal{B}_{i j k l}^{\alpha}\right)$, respectively. We assume that these components satisfy the following classical symmetry and ellipticity conditions: $\mathcal{C}_{i j k l}=\mathcal{C}_{j i k l}=$ $\mathcal{C}_{k l i j} \in W^{1, \infty}\left(\mathbb{R}^{d}\right), \forall i, j, k, l=1, \ldots, d, \exists \alpha_{\mathcal{C}}>0$ such that $\mathcal{C}_{i j k l} \tau_{i j} \tau_{k l} \geq$ $\alpha_{\mathcal{C}} \tau_{i j} \tau_{i j} \forall \tau=\left(\tau_{i j}\right)$ verifying $\tau_{i j}=\tau_{j i}, \forall i, j=1, \ldots, d$, where $\mathcal{C}_{i j k l}=\mathcal{A}_{i j k l}^{\alpha}$, $\mathcal{C}=\mathcal{A}^{\alpha}$ or $\mathcal{C}_{i j k l}=\mathcal{B}_{i j k l}^{\alpha}, \mathcal{C}=\mathcal{B}^{\alpha} \forall i, j, k, l=1, \ldots, d, \alpha=1,2$.

We choose the following state variables (see [22], [10]): the infinitesimal strain tensor $\varepsilon=\left(\varepsilon^{1}, \varepsilon^{2}\right)=\left(\varepsilon\left(\boldsymbol{u}^{1}\right), \varepsilon\left(\boldsymbol{u}^{2}\right)\right)$ in $\Omega^{1} \cup \Omega^{2}$, the normal relative displacement $\left[u_{N}\right]=u_{N}^{1}+u_{N}^{2}-g_{0}$, the tangential relative displacement $\left[\boldsymbol{u}_{T}\right]=$ $\boldsymbol{u}_{T}^{1}-\boldsymbol{u}_{T}^{2}$, and the intensity of adhesion $\beta$ in $\Xi$. We assume that $\boldsymbol{\sigma}^{1} \boldsymbol{n}^{1}=$ $-\boldsymbol{\sigma}^{2} \boldsymbol{n}^{2}$ in $\Xi$, that the normal behaviour is purely elastic for a fixed value of $\beta$ and that the only dissipative processes on the potential contact surfaces are adhesion and friction.

We define $\vartheta: \mathbb{R} \rightarrow \mathbb{R}$ a truncation operator as $\vartheta(s)=-r$ if $s \leq-r$, $\vartheta(s)=s$ if $|s|<r$ and $\vartheta(s)=r$ if $s \geq r$, where $r>0$ is a given characteristic length (see, e.g., [22, 28]). 
We consider the following classical formulation of the dynamic problem coupling adhesion, nonlocal friction and unilateral contact.

Problem $\mathcal{P}_{\boldsymbol{c}}$ : Find $\boldsymbol{u}=\left(\boldsymbol{u}^{1}, \boldsymbol{u}^{2}\right)$ and $\beta$ such that $\boldsymbol{u}(0)=\boldsymbol{u}_{0}, \dot{\boldsymbol{u}}(0)=\boldsymbol{u}_{1}$, $\beta(0)=\beta_{0}$ and

$\ddot{\boldsymbol{u}}^{\alpha}-\operatorname{div} \boldsymbol{\sigma}^{\alpha}\left(\boldsymbol{u}^{\alpha}, \dot{\boldsymbol{u}}^{\alpha}\right)=\boldsymbol{f}^{\alpha}$ in $\left.\Omega^{\alpha} \times\right] 0, T[$,

$\boldsymbol{\sigma}^{\alpha}\left(\boldsymbol{u}^{\alpha}, \dot{\boldsymbol{u}}^{\alpha}\right)=\mathcal{A}^{\alpha} \varepsilon\left(\boldsymbol{u}^{\alpha}\right)+\mathcal{B}^{\alpha} \varepsilon\left(\dot{\boldsymbol{u}}^{\alpha}\right)$ in $\left.\Omega^{\alpha} \times\right] 0, T[$,

$\boldsymbol{u}^{\alpha}=\mathbf{0}$ on $\left.\Gamma_{U}^{\alpha} \times\right] 0, T\left[, \boldsymbol{\sigma}^{\alpha} \boldsymbol{n}^{\alpha}=\boldsymbol{F}^{\alpha}\right.$ on $\left.\Gamma_{F}^{\alpha} \times\right] 0, T[, \alpha=1,2$,

$\boldsymbol{\sigma}^{1} \boldsymbol{n}^{1}+\boldsymbol{\sigma}^{2} \boldsymbol{n}^{2}=\mathbf{0}$ in $\left.\Xi \times\right] 0, T[$,

$\left[u_{N}\right] \leq 0, \sigma_{N}+C_{N}\left[u_{N}\right] \beta^{2} \leq 0,\left(\sigma_{N}+C_{N}\left[u_{N}\right] \beta^{2}\right)\left[u_{N}\right]=0$ in $\left.\Xi \times\right] 0, T[(2.8)$

$\left|\boldsymbol{\sigma}_{T}\right| \leq \mu\left|(\mathcal{R} \boldsymbol{\sigma})_{N}+C_{N}\left[u_{N}\right] \beta^{2}\right|$ in $\left.\Xi \times\right] 0, T[$ and

$$
\begin{aligned}
& \left|\boldsymbol{\sigma}_{T}\right|<\mu\left|(\mathcal{R} \boldsymbol{\sigma})_{N}+C_{N}\left[u_{N}\right] \beta^{2}\right| \Rightarrow\left[\dot{\boldsymbol{u}}_{T}\right]=\mathbf{0}, \\
& \left|\boldsymbol{\sigma}_{T}\right|=\mu\left|(\mathcal{R} \boldsymbol{\sigma})_{N}+C_{N}\left[u_{N}\right] \beta^{2}\right| \Rightarrow \exists \tilde{\lambda} \geq 0,\left[\dot{\boldsymbol{u}}_{T}\right]=-\tilde{\lambda} \boldsymbol{\sigma}_{T},
\end{aligned}
$$

$$
\beta \in[0,1] \text { in } \Xi \times] 0, T[\text { and }
$$

$$
\begin{aligned}
& b \dot{\beta} \geq w \text { if } \beta=0, \\
& \left.b \dot{\beta}=w-C_{N} \vartheta\left(\left[u_{N}\right]^{2}\right) \beta \quad \text { if } \beta \in\right] 0,1[, \\
& b \dot{\beta} \leq w-C_{N} \vartheta\left(\left[u_{N}\right]^{2}\right) \quad \text { if } \beta=1,
\end{aligned}
$$

where $\beta_{0} \in[0,1]$ in $\Xi, C_{N}>0, b>0, w>0, \boldsymbol{\sigma}^{\alpha}=\boldsymbol{\sigma}^{\alpha}\left(\boldsymbol{u}^{\alpha}, \dot{\boldsymbol{u}}^{\alpha}\right), \quad \alpha=1,2$, $\sigma_{N}:=\sigma_{N}^{1}, \boldsymbol{\sigma}_{T}:=\boldsymbol{\sigma}_{T}^{1}, \boldsymbol{\sigma}:=\boldsymbol{\sigma}^{1}, \mu$ is the coefficient of friction and $\mathcal{R}$ is a regularization with good approximation properties which will be presented later.

Note that the healing (rebonding) process is allowed. In the particular case when $\beta=0$, that is the adhesion is totally broken, the classical Signorini's conditions with nonlocal friction are obtained.

\subsection{Variational formulation}

We shall adopt the following notations for some Sobolev spaces and corresponding duality pairings:

$$
\begin{aligned}
& \boldsymbol{H}^{s}:=\left[H^{s}\left(\Omega^{1}\right)\right]^{d} \times\left[H^{s}\left(\Omega^{2}\right)\right]^{d} \forall s \in \mathbb{R}, \\
& \langle\boldsymbol{v}, \boldsymbol{w}\rangle_{-s, s}=\left\langle\boldsymbol{v}^{1}, \boldsymbol{w}^{1}\right\rangle_{\boldsymbol{H}^{-s}\left(\Omega^{1}\right), \boldsymbol{H}^{s}\left(\Omega^{1}\right)}+\left\langle\boldsymbol{v}^{2}, \boldsymbol{w}^{2}\right\rangle_{\boldsymbol{H}^{-s}\left(\Omega^{2}\right), \boldsymbol{H}^{s}\left(\Omega^{2}\right)} \\
& \forall \boldsymbol{v}=\left(\boldsymbol{v}^{1}, \boldsymbol{v}^{2}\right) \in \boldsymbol{H}^{-s}, \forall \boldsymbol{w}=\left(\boldsymbol{w}^{1}, \boldsymbol{w}^{2}\right) \in \boldsymbol{H}^{s} .
\end{aligned}
$$


We define the Hilbert spaces $(\boldsymbol{H},||$.$) with the associated scalar product$ denoted by $(.,),.(\boldsymbol{V},\|\|$.$) with the associated scalar product \left(\right.$ of $\boldsymbol{H}^{1}$ ) denoted by $\langle.,$.$\rangle and the sets \boldsymbol{K}, \Lambda$ as follows:

$$
\begin{aligned}
& \boldsymbol{H}:=\boldsymbol{H}^{0}=\left[L^{2}\left(\Omega^{1}\right)\right]^{d} \times\left[L^{2}\left(\Omega^{2}\right)\right]^{d}, \boldsymbol{V}=\boldsymbol{V}^{1} \times \boldsymbol{V}^{2}, \text { where } \\
& \boldsymbol{V}^{\alpha}=\left\{\boldsymbol{v}^{\alpha} \in\left[H^{1}\left(\Omega^{\alpha}\right)\right]^{d} ; \boldsymbol{v}^{\alpha}=\mathbf{0} \text { a.e. on } \Gamma_{U}^{\alpha}\right\}, \alpha=1,2, \\
& \boldsymbol{K}=\left\{\boldsymbol{v}=\left(\boldsymbol{v}^{1}, \boldsymbol{v}^{2}\right) \in \boldsymbol{V} ;\left[v_{N}\right] \leq 0 \text { a.e. in } \Xi\right\}, \\
& \Lambda=\left\{\lambda \in L^{2}(\Xi) ; \lambda \in[0,1] \text { a.e. in } \Xi\right\} .
\end{aligned}
$$

We assume that $\boldsymbol{F}=\left(\boldsymbol{F}^{1}, \boldsymbol{F}^{2}\right) \in W^{1, \infty}\left(0, T ;\left[L^{2}\left(\Gamma_{F}^{1}\right)\right]^{d}\right) \times W^{1, \infty}\left(0, T ;\left[L^{2}\left(\Gamma_{F}^{2}\right)\right]^{d}\right)$, $\boldsymbol{f}=\left(\boldsymbol{f}^{1}, \boldsymbol{f}^{2}\right) \in W^{1, \infty}\left(0, T ;\left[L^{2}\left(\Omega^{1}\right)\right]^{d}\right) \times W^{1, \infty}\left(0, T ;\left[L^{2}\left(\Omega^{2}\right)\right]^{d}\right), \boldsymbol{u}_{0} \in \boldsymbol{K}$, $\boldsymbol{u}_{1} \in \boldsymbol{V}, \beta_{0} \in \Lambda, \mu \in L^{\infty}(\Xi), \mu \geq 0$ a.e. in $\Xi$.

Let us define two bilinear, continuous and symmetric mappings $a, b$ on $\boldsymbol{H}^{1} \times \boldsymbol{H}^{1} \rightarrow \mathbb{R}$ by

$$
a(\boldsymbol{v}, \boldsymbol{w})=a^{1}\left(\boldsymbol{v}^{1}, \boldsymbol{w}^{1}\right)+a^{2}\left(\boldsymbol{v}^{2}, \boldsymbol{w}^{2}\right), b(\boldsymbol{v}, \boldsymbol{w})=b^{1}\left(\boldsymbol{v}^{1}, \boldsymbol{w}^{1}\right)+b^{2}\left(\boldsymbol{v}^{2}, \boldsymbol{w}^{2}\right)
$$

$\forall \boldsymbol{v}=\left(\boldsymbol{v}^{1}, \boldsymbol{v}^{2}\right), \boldsymbol{w}=\left(\boldsymbol{w}^{1}, \boldsymbol{w}^{2}\right) \in \boldsymbol{H}^{1}$, where, for $\alpha=1,2$,

$a^{\alpha}\left(\boldsymbol{v}^{\alpha}, \boldsymbol{w}^{\alpha}\right)=\int_{\Omega^{\alpha}} \mathcal{A}^{\alpha} \boldsymbol{\varepsilon}\left(\boldsymbol{v}^{\alpha}\right) \cdot \boldsymbol{\varepsilon}\left(\boldsymbol{w}^{\alpha}\right) d x, b^{\alpha}\left(\boldsymbol{v}^{\alpha}, \boldsymbol{w}^{\alpha}\right)=\int_{\Omega^{\alpha}} \mathcal{B}^{\alpha} \boldsymbol{\varepsilon}\left(\boldsymbol{v}^{\alpha}\right) \cdot \boldsymbol{\varepsilon}\left(\boldsymbol{w}^{\alpha}\right) d x$.

Using the previous hypotheses, we consider $\boldsymbol{L}$ as an element of $W^{1, \infty}\left(0, T ; \boldsymbol{H}^{1}\right)$ such that $\forall t \in[0, T]$

$$
\langle\boldsymbol{L}, \boldsymbol{v}\rangle=\sum_{\alpha=1,2} \int_{\Omega^{\alpha}} \boldsymbol{f}^{\alpha} \cdot \boldsymbol{v}^{\alpha} d x+\sum_{\alpha=1,2} \int_{\Gamma_{F}^{\alpha}} \boldsymbol{F}^{\alpha} \cdot \boldsymbol{v}^{\alpha} d s \quad \forall \boldsymbol{v}=\left(\boldsymbol{v}^{1}, \boldsymbol{v}^{2}\right) \in \boldsymbol{H}^{1}
$$

We suppose that $\mathcal{R}:\left[L_{\text {sym }}^{2}\left(\Omega^{1}\right)\right]^{d^{2}} \rightarrow\left[H^{1}\left(\Omega^{1}\right)\right]^{d^{2}}$ is a linear and continuous regularization of $\boldsymbol{\sigma}\left(\boldsymbol{u}^{1}, \boldsymbol{v}^{1}\right)=\boldsymbol{\sigma}^{1}\left(\boldsymbol{u}^{1}, \boldsymbol{v}^{1}\right)$, satisfying $\left(\mathcal{R} \boldsymbol{\sigma}\left(\boldsymbol{u}_{0}^{1}, \boldsymbol{u}_{1}^{1}\right)\right)_{N}=0$ and

$$
\exists C>0, \quad\left\|\mathcal{R} \boldsymbol{\sigma}\left(\boldsymbol{u}^{1}, \boldsymbol{v}^{1}\right)\right\|_{\left[H^{1}\left(\Omega^{1}\right)\right] d^{2}} \leq C\left(\left|\boldsymbol{u}^{1}\right|+\left|\boldsymbol{v}^{1}\right|\right) \quad \forall \boldsymbol{u}^{1}, \boldsymbol{v}^{1} \in \boldsymbol{V}^{1},
$$

where $|$.$| denotes also the norm of \left[L^{2}\left(\Omega^{1}\right)\right]^{d}$. A similar type of regularization can be found in [19] and the same regularization was considered in [9]. 
We define the following mappings:

$$
\begin{aligned}
& c: L^{\infty}(\Xi) \times\left(\boldsymbol{H}^{1}\right)^{2} \rightarrow \mathbb{R}, \quad c(\beta, \boldsymbol{u}, \boldsymbol{v})=\int_{\Xi} C_{N} \vartheta\left(\left[u_{N}\right]\right) \beta^{2}\left(v_{N}^{1}+v_{N}^{2}\right) d \xi, \\
& J: L^{\infty}(\Xi) \times\left(\boldsymbol{H}^{1}\right)^{3} \rightarrow \mathbb{R}, \\
& J(\beta, \boldsymbol{u}, \boldsymbol{v}, \boldsymbol{w})=\int_{\Xi} \mu\left|\left(\mathcal{R} \boldsymbol{\sigma}\left(\boldsymbol{u}^{1}, \boldsymbol{v}^{1}\right)\right)_{N}+C_{N} \vartheta\left(\left[u_{N}\right]\right) \beta^{2}\right|\left|\left[\boldsymbol{w}_{T}\right]\right| d \xi \\
& \forall \beta \in L^{\infty}(\Xi), \quad \forall \boldsymbol{u}=\left(\boldsymbol{u}^{1}, \boldsymbol{u}^{2}\right), \boldsymbol{v}=\left(\boldsymbol{v}^{1}, \boldsymbol{v}^{2}\right), \boldsymbol{w}=\left(\boldsymbol{w}^{1}, \boldsymbol{w}^{2}\right) \in \boldsymbol{H}^{1}, \\
& \gamma: \boldsymbol{H}^{1} \times\left[L^{2}(\Xi)\right]^{2} \rightarrow \mathbb{R}, \quad \gamma(\boldsymbol{u}, \delta, \lambda)=\int_{\Xi} \frac{C_{N}}{b} \vartheta\left(\left[u_{N}\right]^{2}\right) \delta \lambda d \xi \\
& \forall \boldsymbol{u}=\left(\boldsymbol{u}^{1}, \boldsymbol{u}^{2}\right) \in \boldsymbol{H}^{1}, \quad \forall \delta, \lambda \in L^{2}(\Xi) .
\end{aligned}
$$

We denote by $(.,.) \Xi$ the scalar product in $L^{2}(\Xi)$, with the associated norm $|.|_{\Xi}$, and we set $\chi:=\frac{w}{b}$.

We assume also the following compatibility relation on initial conditions: $\exists \boldsymbol{l} \in \boldsymbol{H}$ such that

$$
(\boldsymbol{l}, \boldsymbol{v})+a\left(\boldsymbol{u}_{0}, \boldsymbol{v}\right)+b\left(\boldsymbol{u}_{1}, \boldsymbol{v}\right)+c\left(\beta_{0}, \boldsymbol{u}_{0}, \boldsymbol{v}\right)=\langle\boldsymbol{L}(0), \boldsymbol{v}\rangle \quad \forall \boldsymbol{v} \in \boldsymbol{V}
$$

A variational formulation of the problem $\mathcal{P}_{c}$ is the following.

Problem $\mathcal{P}_{\boldsymbol{v}}:$ Find $\boldsymbol{u} \in W^{1,2}(0, T ; \boldsymbol{V}) \cap C^{1}\left([0, T] ; \boldsymbol{H}^{-1 / 2}\right)$, $\beta \in W^{1, \infty}\left(0, T ; L^{\infty}(\Xi)\right)$ such that $\boldsymbol{u}(0)=\boldsymbol{u}_{0}, \dot{\boldsymbol{u}}(0)=\boldsymbol{u}_{1}$ in $\Omega^{1} \cup \Omega^{2}$, $\beta(0)=\beta_{0}$ in $\Xi, \boldsymbol{u}(t) \in \boldsymbol{K}, \beta(t) \in \Lambda$ for all $\left.t \in\right] 0, T[$ and

$$
\begin{aligned}
& \langle\dot{\boldsymbol{u}}(T), \boldsymbol{v}(T)-\boldsymbol{u}(T)\rangle_{-1 / 2,1 / 2}-\left(\boldsymbol{u}_{1}, \boldsymbol{v}(0)-\boldsymbol{u}_{0}\right)-\int_{0}^{T}(\dot{\boldsymbol{u}}, \dot{\boldsymbol{v}}-\dot{\boldsymbol{u}}) d t \\
& +\int_{0}^{T}\{a(\boldsymbol{u}, \boldsymbol{v}-\boldsymbol{u})+b(\dot{\boldsymbol{u}}, \boldsymbol{v}-\boldsymbol{u})+c(\beta, \boldsymbol{u}, \boldsymbol{v}-\boldsymbol{u})\} d t \\
& +\int_{0}^{T}\{J(\beta, \boldsymbol{u}, \dot{\boldsymbol{u}}, \boldsymbol{v}+\kappa \dot{\boldsymbol{u}}-\boldsymbol{u})-J(\beta, \boldsymbol{u}, \dot{\boldsymbol{u}}, \kappa \dot{\boldsymbol{u}})\} d t \geq \int_{0}^{T}\langle\boldsymbol{L}, \boldsymbol{v}-\boldsymbol{u}\rangle d t \\
& \left.\quad \forall \boldsymbol{v} \in L^{\infty}(0, T ; \boldsymbol{V}) \cap W^{1,2}(0, T ; \boldsymbol{H}) \text { with } \boldsymbol{v}(t) \in \boldsymbol{K} \text { a.e. } t \in\right] 0, T[, \\
& (\dot{\beta}, \lambda-\beta)_{\Xi}+\gamma(\boldsymbol{u}, \beta, \lambda-\beta) \geq(\chi, \lambda-\beta)_{\Xi} \\
& \left.\forall \lambda \in L^{2}\left(0, T ; L^{2}(\Xi)\right) \text { with } \lambda(t) \in \Lambda \text { a.e. } t \in\right] 0, T[
\end{aligned}
$$

where $\kappa>0$. 
The formal equivalence between the variational system (2.13), (2.14) and the classical problem (2.4)-(2.10) can be easily proved by using Green's formula and an integration by parts.

\subsection{An auxiliary penalized problem}

We consider a penalized contact problem that is a dynamic contact problem with normal compliance law, the solution of which is denoted by $\boldsymbol{u}_{\varepsilon}=$ $\left(\boldsymbol{u}_{\varepsilon}^{1}, \boldsymbol{u}_{\varepsilon}^{2}\right), \beta_{\varepsilon}$, where $\varepsilon>0$, verifying the same equations and initial conditions in $\Omega^{1} \cup \Omega^{2}$, the same boundary conditions as in problem $\mathcal{P}_{c}$, excepting the unilateral contact conditions. The new contact conditions in $\Xi \times] 0, T[$ are

$$
\begin{aligned}
& \sigma_{N}^{1}=\sigma_{N}^{2}=-\frac{1}{\varepsilon}\left[u_{\varepsilon N}\right]_{+}-C_{N}\left[u_{\varepsilon N}\right] \beta_{\varepsilon}^{2}, \text { where } r_{+}=\max (r, 0), \\
& \boldsymbol{\sigma}_{T}^{1}\left(\boldsymbol{u}_{\varepsilon}^{1}, \dot{\boldsymbol{u}}_{\varepsilon}^{1}\right)=-\boldsymbol{\sigma}_{T}^{2}\left(\boldsymbol{u}_{\varepsilon}^{2}, \dot{\boldsymbol{u}}_{\varepsilon}^{2}\right), \\
& \left|\boldsymbol{\sigma}_{T}\right| \leq \mu\left|(\mathcal{R} \boldsymbol{\sigma})_{N}+C_{N}\left[u_{\varepsilon N}\right] \beta_{\varepsilon}^{2}\right| \text { and } \\
& \quad\left|\boldsymbol{\sigma}_{T}\right|<\mu\left|(\mathcal{R} \boldsymbol{\sigma})_{N}+C_{N}\left[u_{\varepsilon N}\right] \beta_{\varepsilon}^{2}\right| \Rightarrow\left[\dot{\boldsymbol{u}}_{\varepsilon T}\right]=\mathbf{0}, \\
& \quad\left|\boldsymbol{\sigma}_{T}\right|=\mu\left|(\mathcal{R} \boldsymbol{\sigma})_{N}+C_{N}\left[u_{\varepsilon N}\right] \beta_{\varepsilon}^{2}\right| \Rightarrow \exists \tilde{\lambda} \geq 0,\left[\dot{\boldsymbol{u}}_{\varepsilon T}\right]=-\tilde{\lambda} \boldsymbol{\sigma}_{T}, \\
& \beta_{\varepsilon} \in[0,1] \text { and } \\
& \quad b \dot{\beta}_{\varepsilon} \geq w \text { if } \beta_{\varepsilon}=0, \\
& \left.\quad b \dot{\beta}_{\varepsilon}=w-C_{N} \vartheta\left(\left[u_{\varepsilon N}\right]^{2}\right) \beta_{\varepsilon} \text { if } \beta_{\varepsilon} \in\right] 0,1[, \\
& \quad b \dot{\beta}_{\varepsilon} \leq w-C_{N} \vartheta\left(\left[u_{\varepsilon N}\right]^{2}\right) \text { if } \beta_{\varepsilon}=1 .
\end{aligned}
$$

Let us define the mapping $p_{\varepsilon}: \boldsymbol{V} \times \boldsymbol{V} \rightarrow \mathbb{R}$ by

$$
p_{\varepsilon}(\boldsymbol{v}, \boldsymbol{w})=\frac{1}{\varepsilon} \int_{\Xi} \vartheta\left(\left[v_{N}\right]_{+}\right)\left(w_{N}^{1}+w_{N}^{2}\right) d \xi \quad \forall \boldsymbol{v}, \boldsymbol{w} \in \boldsymbol{V} .
$$

We shall study the following variational formulation of the penalized problem.

Problem $\mathcal{P}_{\varepsilon}$ : Find $\boldsymbol{u}_{\varepsilon} \in W^{2,2}(0, T ; \boldsymbol{H}) \cap W^{1,2}(0, T ; \boldsymbol{V})$ and

$\beta_{\varepsilon} \in W^{1, \infty}\left(0, T ; L^{\infty}(\Xi)\right)$ such that $\boldsymbol{u}_{\varepsilon}(0)=\boldsymbol{u}_{0}, \dot{\boldsymbol{u}}_{\varepsilon}(0)=\boldsymbol{u}_{1}$ in $\Omega^{1} \cup \Omega^{2}$, $\beta_{\varepsilon}(0)=\beta_{0}$ in $\Xi, \beta_{\varepsilon}(s) \in \Lambda$ for all $\left.s \in\right] 0, T[$, and a.e. $t \in] 0, T[$

$$
\begin{aligned}
& \left(\ddot{\boldsymbol{u}}_{\varepsilon}, \boldsymbol{w}-\dot{\boldsymbol{u}}_{\varepsilon}\right)+a\left(\boldsymbol{u}_{\varepsilon}, \boldsymbol{w}-\dot{\boldsymbol{u}}_{\varepsilon}\right)+b\left(\dot{\boldsymbol{u}}_{\varepsilon}, \boldsymbol{w}-\dot{\boldsymbol{u}}_{\varepsilon}\right)+p_{\varepsilon}\left(\boldsymbol{u}_{\varepsilon}, \boldsymbol{w}-\dot{\boldsymbol{u}}_{\varepsilon}\right) \\
& +c\left(\beta_{\varepsilon}, \boldsymbol{u}_{\varepsilon}, \boldsymbol{w}-\dot{\boldsymbol{u}}_{\varepsilon}\right)+J\left(\beta_{\varepsilon}, \boldsymbol{u}_{\varepsilon}, \dot{\boldsymbol{u}}_{\varepsilon}, \boldsymbol{w}\right)-J\left(\beta_{\varepsilon}, \boldsymbol{u}_{\varepsilon}, \dot{\boldsymbol{u}}_{\varepsilon}, \dot{\boldsymbol{u}}_{\varepsilon}\right) \geq\left\langle\boldsymbol{L}, \boldsymbol{w}-\dot{\boldsymbol{u}}_{\varepsilon}\right\rangle \\
& \quad \forall \boldsymbol{w} \in \boldsymbol{V} \\
& \left(\dot{\beta}_{\varepsilon}, \lambda-\beta_{\varepsilon}\right)_{\Xi}+\gamma\left(\boldsymbol{u}_{\varepsilon}, \beta_{\varepsilon}, \lambda-\beta_{\varepsilon}\right) \geq\left(\chi, \lambda-\beta_{\varepsilon}\right)_{\Xi} \quad \forall \lambda \in \Lambda
\end{aligned}
$$




\section{General existence and uniqueness results}

The existence and uniqueness of solutions of problems $\mathcal{P}_{\varepsilon}$ will be obtained by using the following abstract problem.

Let $\left(H_{0},||.\right),\left(V_{0},\|\|.\right)$ and $\left(\Pi_{0},|\cdot|_{\Pi_{0}}\right)$ be three Hilbert spaces with corresponding scalar products denoted by $(.,),.\langle.,$.$\rangle and (., .)_{\Pi_{0}}$, respectively, such that $V_{0}$ is dense in $H_{0}$ with compact imbedding from $V_{0}$ into $H_{0}$ and let $\Lambda_{0}$ be a closed convex set in $\Pi_{0}$. We assume that $0 \in \Lambda_{0}$ and also that $\Lambda_{0}$ is bounded, to simplify the estimates.

We define two bilinear and symmetric forms, $a_{0}, b_{0}: V_{0} \times V_{0} \rightarrow \mathbb{R}$ and the mapping $\gamma_{0}: V_{0} \times \Pi_{0} \times \Pi_{0} \rightarrow \mathbb{R}$ such that

$$
\begin{aligned}
& \exists m_{a}, m_{b}>0 \quad a_{0}(u, v) \leq m_{a}\|u\|\|v\|, \quad b_{0}(u, v) \leq m_{b}\|u\|\|v\|, \\
& \exists A, B>0 \quad a_{0}(v, v) \geq A\|v\|^{2}, \quad b_{0}(v, v) \geq B\|v\|^{2} \quad \forall u, v \in V_{0}, \\
& \forall u \in V_{0} \quad \gamma_{0}(u, \cdot, \cdot) \text { is a bilinear and symmetric form, } \\
& \exists m_{\gamma}>0 \text { such that } \forall u_{1,2} \in V_{0}, \forall \delta_{1,2} \in \Lambda_{0}, \forall \lambda \in \Pi_{0}, \\
& \left|\gamma_{0}\left(u_{1}, \delta_{1}, \lambda\right)-\gamma_{0}\left(u_{2}, \delta_{2}, \lambda\right)\right| \leq m_{\gamma}\left(\left\|u_{1}-u_{2}\right\|+\left|\delta_{1}-\delta_{2}\right|_{\Pi_{0}}\right)|\lambda|_{\Pi_{0}}, \\
& \gamma_{0}(u, \lambda, \lambda) \geq 0 \quad \forall u \in V_{0}, \forall \lambda \in \Pi_{0} .
\end{aligned}
$$

Let $\phi_{0}:[0, T] \times \Lambda_{0} \times V_{0}^{3} \rightarrow \mathbb{R}$ and $\tau_{0}: V_{0} \rightarrow \mathbb{R}$ be two mappings satisfying

$$
\begin{aligned}
& \phi_{0}(t, \lambda, \cdot, \cdot \cdot) \text { and } \tau_{0} \text { are sequentially weakly continuous, } \\
& \phi_{0}\left(t, \lambda, u, v, w_{1}+w_{2}\right) \leq \phi_{0}\left(t, \lambda, u, v, w_{1}\right)+\phi_{0}\left(t, \lambda, u, v, w_{2}\right) \\
& \phi_{0}(t, \lambda, u, v, \theta w)=\theta \phi_{0}(t, \lambda, u, v, w) \\
& \phi_{0}(0,0,0,0, w)=0, \\
& \exists \eta_{0}>0 \text { such that }\left|\tau_{0}(u)\right| \leq \eta_{0}\|u\|, \\
& \quad \forall t \in[0, T], \forall \lambda \in \Lambda_{0}, \forall u, v, w, w_{1,2} \in V_{0}, \forall \theta \geq 0 \\
& \exists \eta_{1}>0 \text { such that } \forall t_{1,2} \in[0, T], \forall \lambda_{1,2} \in \Lambda_{0}, \forall u_{1,2}, v_{1,2}, w \in V_{0}, \\
& \quad\left|\phi_{0}\left(t_{1}, \lambda_{1}, u_{1}, v_{1}, w\right)-\phi_{0}\left(t_{2}, \lambda_{2}, u_{2}, v_{2}, w\right)\right| \\
& \leq \eta_{1}\left(\left|t_{1}-t_{2}\right|+\left|\lambda_{1}-\lambda_{2}\right|_{\Pi_{0}}+\left|\tau_{0}\left(u_{1}-u_{2}\right)\right|+\left|v_{1}-v_{2}\right|\right)\|w\|
\end{aligned}
$$


$\exists \eta_{2}>0$ such that $\forall t_{1,2} \in[0, T], \forall \lambda_{1,2} \in \Lambda_{0}, \forall u_{1,2}, v_{1,2}, w_{1,2} \in V_{0}$,

$$
\begin{aligned}
& \mid \phi_{0}\left(t_{1}, \lambda_{1}, u_{1}, v_{1}, w_{1}\right)-\phi_{0}\left(t_{1}, \lambda_{1}, u_{1}, v_{1}, w_{2}\right) \\
& +\phi_{0}\left(t_{2}, \lambda_{2}, u_{2}, v_{2}, w_{2}\right)-\phi_{0}\left(t_{2}, \lambda_{2}, u_{2}, v_{2}, w_{1}\right) \mid \\
& \leq \eta_{2}\left(\left|t_{1}-t_{2}\right|+\left|\lambda_{1}-\lambda_{2}\right|_{\Pi_{0}}+|| u_{1}-u_{2}||+\left|v_{1}-v_{2}\right|\right)\left\|w_{1}-w_{2}\right\| .
\end{aligned}
$$

We assume that $L_{0} \in W^{1, \infty}\left(0, T ; V_{0}\right), \quad \chi_{0} \in W^{1,2}\left(0, T ; \Pi_{0}\right), u_{0}, u_{1} \in$ $V_{0}, \beta_{0} \in \Lambda_{0}$ and the following compatibility condition: $\exists l_{0} \in H_{0}$ such that $\forall w \in V_{0}$

$$
\left(l_{0}, w\right)+a_{0}\left(u_{0}, w\right)+b_{0}\left(u_{1}, w\right)+\phi_{0}\left(0, \beta_{0}, u_{0}, u_{1}, w\right)=\left\langle L_{0}(0), w\right\rangle .
$$

We consider the following problem.

Problem $\mathcal{Q}$ : Find $u \in W^{2,2}\left(0, T ; H_{0}\right) \cap W^{1,2}\left(0, T ; V_{0}\right), \beta \in W^{1, \infty}\left(0, T ; \Pi_{0}\right)$ such that $u(0)=u_{0}, \dot{u}(0)=u_{1}, \beta(0)=\beta_{0}, \beta(s) \in \Lambda_{0}$ for all $\left.s \in\right] 0, T[$, and a.e. $t \in] 0, T[$

$$
\begin{aligned}
& (\ddot{u}, v-\dot{u})+a_{0}(u, v-\dot{u})+b_{0}(\dot{u}, v-\dot{u}) \\
& \quad+\phi_{0}(t, \beta, u, \dot{u}, v)-\phi_{0}(t, \beta, u, \dot{u}, \dot{u}) \geq\left\langle L_{0}, v-\dot{u}\right\rangle \quad \forall v \in V_{0}, \\
& (\dot{\beta}, \lambda-\beta)_{\Pi_{0}}+\gamma_{0}(u, \beta, \lambda-\beta) \geq\left(\chi_{0}, \lambda-\beta\right)_{\Pi_{0}} \quad \forall \lambda \in \Lambda_{0} .
\end{aligned}
$$

The existence and uniqueness of the solution for problem $\mathcal{Q}$ will be proved by using a result presented in [9], an incremental technique and a fixed point argument.

We define the set

$$
\left.\left.X=\left\{\lambda \in C^{0}\left([0, T] ; \Pi_{0}\right) ; \lambda(0)=\beta_{0}, \lambda(t) \in \Lambda_{0} \forall t \in\right] 0, T\right]\right\},
$$

where the Banach space $C^{0}\left([0, T] ; \Pi_{0}\right)$ is endowed with the norm

$$
\|\lambda\|_{k}=\max _{t \in[0, T]}\left[\exp (-k t)|\lambda(t)|_{\Pi_{0}}\right] \text { for all } \lambda \in C^{0}\left([0, T] ; \Pi_{0}\right), k \geq 0 .
$$

Using the Theorem 3.2 of [9], which for every $\beta \in X$ clearly can be applied with minor modifications to (3.14) with the initial conditions $u_{0}, u_{1}$, we have the following result.

Lemma 3.1. For each $\beta \in X$ there exists a unique $u_{\beta} \in W^{2,2}\left(0, T ; H_{0}\right) \cap$ $W^{1,2}\left(0, T ; V_{0}\right)$ solution of the inequality (3.14) such that $u_{\beta}(0)=u_{0}, \dot{u}_{\beta}(0)=$ $u_{1}$. 
Lemma 3.2. Let $\beta_{1}, \beta_{2} \in X$ and let $u_{\beta_{1}}, u_{\beta_{2}}$ be the corresponding solutions of (3.14) with the same initial conditions $u_{0}, u_{1}$, respectively. Then there exists a constant $C_{1}>0$, independent of $\beta_{1}, \beta_{2}, u_{\beta_{1}}, u_{\beta_{2}}$, such that for all $t \in[0, T]$

$$
\left|\dot{u}_{\beta_{1}}(t)-\dot{u}_{\beta_{2}}(t)\right|^{2}+\left\|u_{\beta_{1}}(t)-u_{\beta_{2}}(t)\right\|^{2} \leq C_{1} \int_{0}^{t}\left|\beta_{1}(s)-\beta_{2}(s)\right|_{\Pi_{0}}^{2} d s .
$$

Proof. Let $u_{\beta_{1}}, u_{\beta_{2}}$ be the solutions of (3.14) corresponding to $\beta_{1}, \beta_{2} \in X$. Taking in each inequality $v=\dot{u}_{\beta_{2}}$ and $v=\dot{u}_{\beta_{1}}$, respectively, for a.e. $\left.s \in\right] 0, T[$ we have

$$
\begin{aligned}
& \left(\ddot{u}_{\beta_{1}}-\ddot{u}_{\beta_{2}}, \dot{u}_{\beta_{1}}-\dot{u}_{\beta_{2}}\right)+a_{0}\left(u_{\beta_{1}}-u_{\beta_{2}}, \dot{u}_{\beta_{1}}-\dot{u}_{\beta_{2}}\right)+b_{0}\left(\dot{u}_{\beta_{1}}-\dot{u}_{\beta_{2}}, \dot{u}_{\beta_{1}}-\dot{u}_{\beta_{2}}\right) \\
& \quad \leq \phi_{0}\left(s, \beta_{1}, u_{\beta_{1}}, \dot{u}_{\beta_{1}}, \dot{u}_{\beta_{2}}\right)-\phi_{0}\left(s, \beta_{1}, u_{\beta_{1}}, \dot{u}_{\beta_{1}}, \dot{u}_{\beta_{1}}\right) \\
& \quad+\phi_{0}\left(s, \beta_{2}, u_{\beta_{2}}, \dot{u}_{\beta_{2}}, \dot{u}_{\beta_{1}}\right)-\phi_{0}\left(s, \beta_{2}, u_{\beta_{2}}, \dot{u}_{\beta_{2}}, \dot{u}_{\beta_{2}}\right) \\
& \quad \leq \eta_{2}\left(\left|\beta_{1}-\beta_{2}\right|_{\Pi_{0}}+\left\|u_{\beta_{1}}-u_{\beta_{2}}\right\|+\left|\dot{u}_{\beta_{1}}-\dot{u}_{\beta_{2}}\right|\right)\left\|\dot{u}_{\beta_{1}}-\dot{u}_{\beta_{2}}\right\|
\end{aligned}
$$

where the second inequality follows by (3.12). For all $t \in[0, T]$, as the solutions $u_{\beta_{1}}, u_{\beta_{2}}$ verify the same initial conditions, by integrating between 0 and $t$ we obtain

$$
\begin{aligned}
& \frac{1}{2}\left|\dot{u}_{\beta_{1}}(t)-\dot{u}_{\beta_{2}}(t)\right|^{2}+\frac{1}{2} a_{0}\left(u_{\beta_{1}}(t)-u_{\beta_{2}}(t), u_{\beta_{1}}(t)-u_{\beta_{2}}(t)\right) \\
& +\int_{0}^{t} b_{0}\left(\dot{u}_{\beta_{1}}-\dot{u}_{\beta_{2}}, \dot{u}_{\beta_{1}}-\dot{u}_{\beta_{2}}\right) d s \leq \eta_{2} \int_{0}^{t}\left|\beta_{1}-\beta_{2}\right|_{\Pi_{0}}\left\|\dot{u}_{\beta_{1}}-\dot{u}_{\beta_{2}}\right\| d s \\
& \quad+\eta_{2} \int_{0}^{t}\left(\left\|u_{\beta_{1}}-u_{\beta_{2}}\right\|\left\|\dot{u}_{\beta_{1}}-\dot{u}_{\beta_{2}}\right\|+\left|\dot{u}_{\beta_{1}}-\dot{u}_{\beta_{2}}\right|\left\|\dot{u}_{\beta_{1}}-\dot{u}_{\beta_{2}}\right\|\right) d s .
\end{aligned}
$$

Using Young's inequalities for the last three terms with an appropriate constant, and $V_{0}$ - ellipticity of $a_{0}, b_{0}$, it follows that

$$
\begin{aligned}
& \frac{1}{2}\left|\dot{u}_{\beta_{1}}(t)-\dot{u}_{\beta_{2}}(t)\right|^{2}+\frac{A}{2}\left\|u_{\beta_{1}}(t)-u_{\beta_{2}}(t)\right\|^{2}+\frac{B}{2} \int_{0}^{t}\left\|\dot{u}_{\beta_{1}}-\dot{u}_{\beta_{2}}\right\|^{2} d s \\
& \quad \leq \frac{3 \eta_{2}^{2}}{2 B} \int_{0}^{t}\left|\beta_{1}-\beta_{2}\right|_{\Pi_{0}}^{2} d s+\frac{3 \eta_{2}^{2}}{2 B} \int_{0}^{t}\left(\left\|u_{\beta_{1}}-u_{\beta_{2}}\right\|^{2}+\left|\dot{u}_{\beta_{1}}-\dot{u}_{\beta_{2}}\right|^{2}\right) d s .
\end{aligned}
$$

By Gronwall's lemma we obtain the estimate (3.16).

Now, for every element $u \in W^{1,2}\left(0, T ; V_{0}\right)$, we consider the inequality (3.15) with the initial condition $\beta_{0}$, the solution of which is denoted by $\beta_{u}$. 
The existence and uniqueness results for this parabolic inequality follow by classical references, see, e.g., [5] or [2], but we prefer to present a direct proof based on an incremental technique, for the convenience of the reader.

Proposition 3.3. For each $u \in W^{1,2}\left(0, T ; V_{0}\right)$ there exists a unique $\beta_{u} \in$ $X \cap W^{1, \infty}\left(0, T ; \Pi_{0}\right)$, solution of the inequality (3.15).

Proof. We consider an incremental formulation obtained by using an implicit time discretization scheme for (3.15). For $n \in \mathbb{N}^{*}$, we set $\Delta t:=$ $T / n$ and $t_{i}:=i \Delta t, i=0,1, \ldots, n$, and $\beta^{0}:=\beta_{0}$. If $\psi$ is a continuous function of $t \in[0, T]$ valued in some vector space, we use the notations $\psi^{i}:=\psi\left(t_{i}\right)$ and if $\left(\theta^{i}\right)_{i \in\{0,1, \ldots, n\}}$ are elements of some vector space, then for all $i \in\{0,1, \ldots, n-1\}$ we set $\Delta \theta^{i}:=\theta^{i+1}-\theta^{i}$. We then approximate (3.15) using the sequence of following incremental problems: for $i=0,1, \ldots, n-1$, find $\beta^{i+1} \in \Lambda_{0}$ such that

$$
\left(\frac{\Delta \beta^{i}}{\Delta t}, \lambda-\beta^{i+1}\right)_{\Pi_{0}}+\gamma_{0}\left(u^{i+1}, \beta^{i+1}, \lambda-\beta^{i+1}\right) \geq\left(\chi_{0}^{i+1}, \lambda-\beta^{i+1}\right)_{\Pi_{0}} \forall \lambda \in \Lambda_{0} .
$$

As the previous inequality can be written in the equivalent form

$$
\begin{aligned}
& \left(\beta^{i+1}, \lambda-\beta^{i+1}\right)_{\Pi_{0}}+\Delta t \gamma_{0}\left(u^{i+1}, \beta^{i+1}, \lambda-\beta^{i+1}\right) \\
& \geq\left(\Delta t \chi_{0}^{i+1}+\beta^{i}, \lambda-\beta^{i+1}\right)_{\Pi_{0}} \forall \lambda \in \Lambda_{0},
\end{aligned}
$$

which is an elliptic variational inequality of the first kind that contains the scalar product in $\Pi_{0}$ and $\gamma_{0}$ satisfying (3.3)-(3.5), by a classical result it follows that there exists a unique solution $\beta^{i+1}$ of (3.17).

Taking in (3.17) $\lambda=\beta^{i}$, using (3.4) and Cauchy-Schwarz inequality, we have

$$
\begin{aligned}
\left(\frac{\Delta \beta^{i}}{\Delta t}, \Delta \beta^{i}\right)_{\Pi_{0}} & \leq-\gamma_{0}\left(u^{i+1}, \beta^{i+1}, \Delta \beta^{i}\right)+\left(\chi_{0}^{i+1}, \Delta \beta^{i}\right)_{\Pi_{0}} \\
& \leq m_{\gamma}\left(\left\|u^{i+1}\right\|+\left|\beta^{i+1}\right|_{\Pi_{0}}\right)\left|\Delta \beta^{i}\right|_{\Pi_{0}}+\left|\chi_{0}^{i+1}\right|_{\Pi_{0}}\left|\Delta \beta^{i}\right|_{\Pi_{0}} .
\end{aligned}
$$

Hence,

$$
\frac{\left|\Delta \beta^{i}\right|_{\Pi_{0}}}{\Delta t} \leq m_{\gamma}\left(\left\|u^{i+1}\right\|+\left|\beta^{i+1}\right|_{\Pi_{0}}\right)+\left|\chi_{0}^{i+1}\right|_{\Pi_{0}}, \text { for } i=0,1, \ldots, n-1 .
$$

Let us define the following functions:

$$
\begin{aligned}
& \beta_{n}(0)=\hat{\beta}_{n}(0)=\beta^{0}, u_{n}(0)=u^{0}, \chi_{n}(0)=\chi_{0}^{0} \text { and } \\
& \left.\forall i \in\{0,1, \ldots, n-1\}, \forall t \in] t_{i}, t_{i+1}\right], \\
& \beta_{n}(t)=\beta^{i+1}, \hat{\beta}_{n}(t)=\beta^{i}+\left(t-t_{i}\right) \frac{\Delta \beta^{i}}{\Delta t}, \\
& u_{n}(t)=u^{i+1}, \chi_{n}(t)=\chi_{0}^{i+1} .
\end{aligned}
$$


Then $\beta_{n} \in L^{2}\left(0, T ; \Pi_{0}\right), \hat{\beta}_{n} \in W^{1,2}\left(0, T ; \Pi_{0}\right), u_{n} \rightarrow u$ in $L^{2}\left(0, T ; V_{0}\right)$, $\chi_{n} \rightarrow \chi_{0}$ in $L^{2}\left(0, T ; \Pi_{0}\right)$ and $\beta_{n}(t) \in \Lambda_{0}, u_{n}(t) \rightarrow u(t)$ in $V_{0}, \chi_{n}(t) \rightarrow$ $\chi_{0}(t)$ in $\Pi_{0} \quad \forall t \in[0, T]$. Since $u \in W^{1,2}\left(0, T ; V_{0}\right) \subset C^{0}\left([0, T] ; V_{0}\right)$, $\chi_{0} \in W^{1,2}\left(0, T ; \Pi_{0}\right) \subset C^{0}\left([0, T] ; \Pi_{0}\right)$, it follows that $\left(\left\|u_{n}(t)\right\|\right)_{n \in \mathbb{N}^{*}}$ and $\left(\left|\chi_{n}(t)\right|_{\Pi_{0}}\right)_{n \in \mathbb{N}^{*}}$ are bounded by constants independent of $t \in[0, T]$.

Also, for all $n \in \mathbb{N}^{*}$ we have

$$
\left.\left|\beta_{n}(t)-\hat{\beta}_{n}(t)\right|_{\Pi_{0}} \leq \frac{T}{n}\left|\frac{d}{d t} \hat{\beta}_{n}(t)\right|_{\Pi_{0}} \text { for a.e. } t \in\right] 0, T[
$$

and the estimates (3.18) $, i=0,1, \ldots, n-1$, imply the following relation:

$$
\left.\left|\frac{d}{d t} \hat{\beta}_{n}(t)\right|_{\Pi_{0}} \leq m_{\gamma}\left(\left\|u_{n}(t)\right\|+\left|\beta_{n}(t)\right|_{\Pi_{0}}\right)+\left|\chi_{n}(t)\right|_{\Pi_{0}} \text { for a.e. } t \in\right] 0, T[.
$$

Thus, there exists a constant $C_{2}>0$ satisfying

$$
\left\|\hat{\beta}_{n}\right\|_{W^{1, \infty}\left(0, T ; \Pi_{0}\right)} \leq C_{2} \text { for all } n \in \mathbb{N}^{*}
$$

so that there exists a subsequence, still denoted by $\left(\hat{\beta}_{n}\right)_{n}$, and an element $\beta_{u} \in W^{1, \infty}\left(0, T ; \Pi_{0}\right)$ such that

$$
\hat{\beta}_{n} \rightarrow^{*} \beta_{u} \text { in } W^{1, \infty}\left(0, T ; \Pi_{0}\right),
$$

and, by (3.20),

$$
\beta_{n} \rightarrow^{*} \beta_{u} \quad \text { in } L^{\infty}\left(0, T ; \Pi_{0}\right) .
$$

Applying a diagonal process, see, e.g., [7], it follows from (3.22), (3.20) that, up to a subsequence,

$$
\beta_{n}(t) \rightarrow \beta_{u}(t) \quad \text { in } \Pi_{0} \quad \forall t \in[0, T],
$$

which implies that $\beta_{u}(0)=\beta_{0}$ and $\beta_{u}(t) \in \Lambda_{0}$ for all $\left.t \in\right] 0, T[$.

We shall prove that the limit $\beta_{u}$ is a solution of the inequality (3.15). The sequence of inequalities (3.17), $i=0,1, \ldots, n-1$, is equivalent to the following incremental formulation: $\beta_{n}(t) \in \Lambda_{0}$ for all $t \in[0, T]$ and

$$
\begin{aligned}
\left(\frac{d}{d t} \hat{\beta}_{n}(t), \lambda\right. & \left.-\beta_{n}(t)\right)_{\Pi_{0}}+\gamma_{0}\left(u_{n}(t), \beta_{n}(t), \lambda-\beta_{n}(t)\right) \\
& \left.\geq\left(\chi_{n}(t), \lambda-\beta_{n}(t)\right)_{\Pi_{0}} \quad \forall \lambda \in \Lambda_{0}, \text { for a.e. } t \in\right] 0, T[
\end{aligned}
$$


Integrating both sides of (3.25) over $[0, T]$ it follows that for all $\lambda \in$ $L^{2}\left(0, T ; \Pi_{0}\right)$ such that $\lambda(t) \in \Lambda_{0}$ for a.e. $\left.t \in\right] 0, T[$,

$$
\begin{gathered}
\int_{0}^{T}\left(\frac{d}{d t} \hat{\beta}_{n}(t), \lambda(t)-\beta_{n}(t)\right)_{\Pi_{0}} d t+\int_{0}^{T} \gamma_{0}\left(u_{n}(t), \beta_{n}(t), \lambda(t)-\beta_{n}(t)\right) d t \\
\geq \int_{0}^{T}\left(\chi_{n}(t), \lambda(t)-\beta_{n}(t)\right)_{\Pi_{0}} d t
\end{gathered}
$$

Using the properties of the corresponding sequences, of the scalar product and of $\gamma_{0}$, we have

$$
\begin{aligned}
& \int_{0}^{T}\left(\frac{d}{d t} \hat{\beta}_{n}(t), \beta_{n}(t)\right)_{\Pi_{0}} d t \geq \frac{1}{2}\left[\left(\beta_{n}(T), \beta_{n}(T)\right)_{\Pi_{0}}-\left(\beta_{0}, \beta_{0}\right)_{\Pi_{0}}\right], \\
& \lim _{n \rightarrow \infty}\left|\int_{0}^{T}\left[\gamma_{0}\left(u_{n}(t), \beta_{n}(t), \beta_{n}(t)\right)-\gamma_{0}\left(u(t), \beta_{n}(t), \beta_{n}(t)\right)\right] d t\right|=0 .
\end{aligned}
$$

Therefore, by passing to the limit we obtain

$$
\begin{aligned}
& \liminf _{n \rightarrow \infty} \int_{0}^{T}\left(\frac{d}{d t} \hat{\beta}_{n}(t), \beta_{n}(t)\right)_{\Pi_{0}} d t \geq \int_{0}^{T}\left(\dot{\beta}_{u}(t), \beta_{u}(t)\right)_{\Pi_{0}} d t \\
& \liminf _{n \rightarrow \infty} \int_{0}^{T} \gamma_{0}\left(u_{n}(t), \beta_{n}(t), \beta_{n}(t)\right) d t \geq \int_{0}^{T} \gamma_{0}\left(u(t), \beta_{u}(t), \beta_{u}(t)\right) d t .
\end{aligned}
$$

Since we clearly have

$$
\begin{aligned}
& \lim _{n \rightarrow \infty} \int_{0}^{T}\left(\frac{d}{d t} \hat{\beta}_{n}(t), \lambda(t)\right)_{\Pi_{0}} d t=\int_{0}^{T}\left(\dot{\beta}_{u}(t), \lambda(t)\right)_{\Pi_{0}} d t \\
& \lim _{n \rightarrow \infty} \int_{0}^{T} \gamma_{0}\left(u_{n}(t), \beta_{n}(t), \lambda(t)\right) d t \\
& \quad=\lim _{n \rightarrow \infty} \int_{0}^{T}\left[\gamma_{0}\left(u_{n}(t), \beta_{n}(t), \lambda(t)\right)-\gamma_{0}\left(u(t), \beta_{n}(t), \lambda(t)\right)\right] d t \\
& \quad+\lim _{n \rightarrow \infty} \int_{0}^{T} \gamma_{0}\left(u(t), \beta_{n}(t), \lambda(t)\right) d t=\int_{0}^{T} \gamma_{0}\left(u(t), \beta_{u}(t), \lambda(t)\right) d t, \\
& \lim _{n \rightarrow \infty} \int_{0}^{T}\left(\chi_{n}(t), \lambda(t)-\beta_{n}(t)\right)_{\Pi_{0}} d t=\int_{0}^{T}\left(\chi_{0}(t), \lambda(t)-\beta_{u}(t)\right)_{\Pi_{0}} d t
\end{aligned}
$$


finally by passing to the limit in (3.26), we obtain that for all $\lambda \in L^{2}\left(0, T ; \Pi_{0}\right)$ such that $\lambda(t) \in \Lambda_{0}$ for a.e. $\left.t \in\right] 0, T[$

$$
\begin{gathered}
\int_{0}^{T}\left(\dot{\beta}_{u}(t), \lambda(t)-\beta_{u}(t)\right)_{\Pi_{0}} d t+\int_{0}^{T} \gamma_{0}\left(u(t), \beta_{u}(t), \lambda(t)-\beta_{u}(t)\right) d t \\
\geq \int_{0}^{T}\left(\chi_{0}(t), \lambda(t)-\beta_{u}(t)\right)_{\Pi_{0}} d t .
\end{gathered}
$$

By Lebesgue's theorem, it follows that $\beta_{u}$ is a solution of the parabolic inequality (3.15).

In order to show the uniqueness of $\beta_{u}$, let $\beta_{1}, \beta_{2}$ be two solutions of (3.15) corresponding to $u \in W^{1,2}\left(0, T ; V_{0}\right)$. Taking in each inequality $\lambda=\beta_{2}$ and $\lambda=\beta_{1}$, respectively, we derive that for a.e. $\left.s \in\right] 0, T[$

$$
\left(\dot{\beta}_{1}(s)-\dot{\beta}_{2}(s), \beta_{1}(s)-\beta_{2}(s)\right)_{\Pi_{0}}+\gamma_{0}\left(u(s), \beta_{1}(s)-\beta_{2}(s), \beta_{1}(s)-\beta_{2}(s)\right) \leq 0 .
$$

Using that $\beta_{1}, \beta_{2}$ satisfy the same initial condition, for all $\left.t \in\right] 0, T[$ by integrating over $[0, t]$ we have

$$
\frac{1}{2}\left|\beta_{1}(t)-\beta_{2}(t)\right|_{\Pi_{0}}^{2}+\int_{0}^{t} \gamma_{0}\left(u(s), \beta_{1}(s)-\beta_{2}(s), \beta_{1}(s)-\beta_{2}(s)\right) d s \leq 0,
$$

which implies that $\beta_{1}=\beta_{2}$.

Lemma 3.4. Let $u_{1}, u_{2} \in W^{1,2}\left(0, T ; V_{0}\right)$ and let $\beta_{u_{1}}, \beta_{u_{2}} \in X$ be the corresponding solutions of (3.15) with the same initial condition $\beta_{0}$, respectively. Then there exists a constant $C_{3}>0$, independent of $u_{1}, u_{2}, \beta_{u_{1}}, \beta_{u_{2}}$, such that for all $t \in[0, T]$

$$
\left|\beta_{u_{1}}(t)-\beta_{u_{2}}(t)\right|_{\Pi_{0}}^{2} \leq C_{3} \int_{0}^{t}\left\|u_{1}(s)-u_{2}(s)\right\|^{2} d s .
$$

Proof. Let $\beta_{u_{1}}, \beta_{u_{2}}$ be the solutions of (3.15) corresponding to $u_{1}, u_{2}$. Taking in each inequality $\lambda=\beta_{u_{2}}, \lambda=\beta_{u_{1}}$, respectively, for all $\left.t \in\right] 0, T$, by 
integrating over $[0, t]$, using (3.4) and some elementary inequality we have

$$
\begin{aligned}
\frac{1}{2} \mid \beta_{u_{1}}(t) & -\left.\beta_{u_{2}}(t)\right|_{\Pi_{0}} ^{2} \\
& \leq \int_{0}^{t}\left[\gamma_{0}\left(u_{2}, \beta_{u_{2}}, \beta_{u_{1}}-\beta_{u_{2}}\right)-\gamma_{0}\left(u_{1}, \beta_{u_{1}}, \beta_{u_{1}}-\beta_{u_{2}}\right)\right] d s \\
& =\int_{0}^{t}\left[\gamma_{0}\left(u_{2}, \beta_{u_{2}}, \beta_{u_{1}}-\beta_{u_{2}}\right)-\gamma_{0}\left(u_{2}, \beta_{u_{1}}, \beta_{u_{1}}-\beta_{u_{2}}\right)\right] d s \\
& +\int_{0}^{t}\left[\gamma_{0}\left(u_{2}, \beta_{u_{1}}, \beta_{u_{1}}-\beta_{u_{2}}\right)-\gamma_{0}\left(u_{1}, \beta_{u_{1}}, \beta_{u_{1}}-\beta_{u_{2}}\right)\right] d s \\
& \leq m_{\gamma} \int_{0}^{t}\left|\beta_{u_{1}}-\beta_{u_{2}}\right|_{\Pi_{0}}^{2} d s+m_{\gamma} \int_{0}^{t}\left\|u_{1}-u_{2}\right\|\left|\beta_{u_{1}}-\beta_{u_{2}}\right| \Pi_{0} d s \\
& \leq \frac{m_{\gamma}}{2} \int_{0}^{t}\left\|u_{1}(s)-u_{2}(s)\right\|^{2} d s+\frac{3 m_{\gamma}}{2} \int_{0}^{t}\left|\beta_{u_{1}}(s)-\beta_{u_{2}}(s)\right|_{\Pi_{0}}^{2} d s,
\end{aligned}
$$

where, to simplify, the variable $s$ was omitted in some relations. By Gronwall's lemma we obtain the estimate (3.27).

Now we can prove the following existence and uniqueness result.

Theorem 3.5. Assume that (3.1)-(3.12) and the compatibility condition (3.13) hold. Then there exists a unique solution of the problem $\mathcal{Q}$.

Proof. For every $\beta \in X$ let $u_{\beta} \in W^{2,2}\left(0, T ; H_{0}\right) \cap W^{1,2}\left(0, T ; V_{0}\right)$ be the solution of the inequality (3.14) corresponding to $\beta$ such that $u_{\beta}(0)=u_{0}$, $\dot{u}_{\beta}(0)=u_{1}$ and let $\beta_{u_{\beta}} \in X \cap W^{1, \infty}\left(0, T ; \Pi_{0}\right)$ be the solution of the inequality (3.15) corresponding to $u_{\beta}$. We define the mapping $\mathcal{T}: X \rightarrow X$ by $\forall \beta \in X \quad \mathcal{T} \beta=\beta_{u_{\beta}}$ and we will prove that $\mathcal{T}: X \rightarrow X$ has a unique fixed point, which is equally the solution of the problem $\mathcal{Q}$.

For all $\beta_{1}, \beta_{2} \in X$, for all $t \in[0, T]$, using (3.27) and (3.16), we have

$$
\begin{aligned}
\mid \mathcal{T} \beta_{1}(t) & -\left.\mathcal{T} \beta_{2}(t)\right|_{\Pi_{0}} ^{2} \leq C_{3} \int_{0}^{t}\left\|u_{\beta_{1}}(s)-u_{\beta_{2}}(s)\right\|^{2} d s \\
& \leq C_{1} C_{3} \int_{0}^{t}\left(\int_{0}^{s} \exp (-2 k r) \exp (2 k r)\left|\beta_{1}(r)-\beta_{2}(r)\right|_{\Pi_{0}}^{2} d r\right) d s \\
& \leq C_{1} C_{3}\left\|\beta_{1}-\beta_{2}\right\|_{k}^{2} \int_{0}^{t} \frac{\exp (2 k s)}{2 k} d s \\
& \leq \frac{C_{1} C_{3}}{4 k^{2}} \cdot \exp (2 k t)\left\|\beta_{1}-\beta_{2}\right\|_{k}^{2} .
\end{aligned}
$$


Then

$$
\begin{gathered}
\left\|\mathcal{T} \beta_{1}-\mathcal{T} \beta_{2}\right\|_{k}=\max _{t \in[0, T]}\left[\exp (-k t)\left|\mathcal{T} \beta_{1}(t)-\mathcal{T} \beta_{2}(t)\right|_{\Pi_{0}}\right] \\
\leq \frac{\sqrt{C_{1} C_{3}}}{2 k}\left\|\beta_{1}-\beta_{2}\right\|_{k} .
\end{gathered}
$$

Hence, for all $\beta_{1}, \beta_{2} \in X$

$$
\left\|\mathcal{T} \beta_{1}-\mathcal{T} \beta_{2}\right\|_{k} \leq \frac{\sqrt{C_{1} C_{3}}}{2 k}\left\|\beta_{1}-\beta_{2}\right\|_{k}
$$

so that if $k$ is sufficiently large it follows that $\mathcal{T}$ is a contraction and its fixed point is the solution of the problem $\mathcal{Q}$.

\section{Approximation and existence of variational solutions}

Now, the previous general results are applied to analyse the penalized and the unilateral contact problems.

\subsection{Existence and uniqueness of penalized solutions}

We prove the following existence and uniqueness result for the penalized problem.

Theorem 4.1. There exists a unique solution to the problem $\mathcal{P}_{\varepsilon}$.

Proof. We apply Theorem 3.5 to $H_{0}=\boldsymbol{H}, V_{0}=\boldsymbol{V}, \Pi_{0}=L^{2}(\Xi), \Lambda_{0}=\Lambda$, $u_{0}=\boldsymbol{u}_{0}, u_{1}=\boldsymbol{u}_{1}, a_{0}=a, b_{0}=b, L_{0}=\boldsymbol{L},(., .)_{\Pi_{0}}=(.,.) \Xi,|\cdot|_{\Pi_{0}}=|.|_{\Xi}, \gamma_{0}=$ $\gamma, \chi_{0}=\chi$ and

$$
\begin{gathered}
\phi_{0}(t, \lambda, \boldsymbol{u}, \boldsymbol{v}, \boldsymbol{w})=p_{\varepsilon}(\boldsymbol{u}, \boldsymbol{w})+c(\lambda, \boldsymbol{u}, \boldsymbol{w})+J(\lambda, \boldsymbol{u}, \boldsymbol{v}, \boldsymbol{w}) \\
\forall t \in[0, T], \forall \lambda \in L^{2}(\Xi), \forall \boldsymbol{u}, \boldsymbol{v}, \boldsymbol{w} \in \boldsymbol{V} .
\end{gathered}
$$

As meas $\left(\Gamma_{U}^{\alpha}\right)>0$, the ellipticity property of the coefficients $\mathcal{A}_{i j k l}^{\alpha}, \mathcal{B}_{i j k l}^{\alpha}$ and the Korn's inequality imply that there exist $A^{\alpha}, B^{\alpha}>0$ such that

$$
a^{\alpha}\left(\boldsymbol{v}^{\alpha}, \boldsymbol{v}^{\alpha}\right) \geq A^{\alpha}\left\|\boldsymbol{v}^{\alpha}\right\|_{\boldsymbol{V}^{\alpha}}^{2}, b^{\alpha}\left(\boldsymbol{v}^{\alpha}, \boldsymbol{v}^{\alpha}\right) \geq B^{\alpha}\left\|\boldsymbol{v}^{\alpha}\right\|_{\boldsymbol{V}^{\alpha}}^{2} \forall \boldsymbol{v}^{\alpha} \in \boldsymbol{V}^{\alpha}, \alpha=1,2,
$$

and we obtain

$$
a(\boldsymbol{v}, \boldsymbol{v}) \geq A\|\boldsymbol{v}\|^{2}, \quad b(\boldsymbol{v}, \boldsymbol{v}) \geq B\|\boldsymbol{v}\|^{2} \forall \boldsymbol{v} \in \boldsymbol{V},
$$


where $A=\min \left(A^{1}, A^{2}\right), B=\min \left(B^{1}, B^{2}\right)$.

For all $\boldsymbol{u}_{1,2} \in \boldsymbol{V}, \delta_{1,2} \in \Lambda, \lambda \in L^{2}(\Xi)$, we have the following relations:

$$
\begin{aligned}
& \left|\gamma\left(\boldsymbol{u}_{1}, \delta_{1}, \lambda\right)-\gamma\left(\boldsymbol{u}_{2}, \delta_{2}, \lambda\right)\right|=\left|\frac{C_{N}}{b} \int_{\Xi}\left(\vartheta\left(\left[u_{1 N}\right]^{2}\right) \delta_{1}-\vartheta\left(\left[u_{2 N}\right]^{2}\right) \delta_{2}\right) \lambda d \xi\right| \\
& \quad=\left|\frac{C_{N}}{b} \int_{\Xi}\left[\left(\vartheta\left(\left[u_{1 N}\right]\right)^{2}-\vartheta\left(\left[u_{2 N}\right]\right)^{2}\right) \delta_{1}+\vartheta\left(\left[u_{2 N}\right]^{2}\right)\left(\delta_{1}-\delta_{2}\right)\right] \lambda d \xi\right| \\
& \quad \leq \frac{C_{N}}{b} \int_{\Xi}\left[2 r\left|\vartheta\left(\left[u_{1 N}\right]\right)-\vartheta\left(\left[u_{2 N}\right]\right)\right|+r^{2}\left|\delta_{1}-\delta_{2}\right|\right]|\lambda| d \xi \\
& \quad \leq \max \left(2 r, r^{2}\right) \frac{C_{N}}{b} \int_{\Xi}\left(\left|\left[u_{1 N}\right]-\left[u_{2 N}\right]\right|+\left|\delta_{1}-\delta_{2}\right|\right)|\lambda| d \xi \\
& \quad=\max \left(2 r, r^{2}\right) \frac{C_{N}}{b} \int_{\Xi}\left(\left|u_{1 N}^{1}+u_{1 N}^{2}-u_{2 N}^{1}-u_{2 N}^{2}\right|+\left|\delta_{1}-\delta_{2}\right|\right)|\lambda| d \xi \\
& \quad \leq m_{\gamma}\left(\left\|\boldsymbol{u}_{1}-\boldsymbol{u}_{2}\right\|+\left|\delta_{1}-\delta_{2}\right| \Xi\right)|\lambda|_{\Xi} .
\end{aligned}
$$

Thus, (3.2) and (3.4) are satisfied. We can also easily verify (3.1), (3.3) and (3.5).

For all $\lambda \in L^{2}(\Xi), \boldsymbol{u} \in \boldsymbol{V}$, the mappings $p_{\varepsilon}(\boldsymbol{u}, \cdot), c(\lambda, \boldsymbol{u}, \cdot)$ are linear on $\boldsymbol{V}$ and the mapping $J(\lambda, \boldsymbol{u}, \boldsymbol{v}, \cdot)$ is a semi-norm on $\boldsymbol{V}$, which imply that $\phi_{0}$ satisfies conditions (3.7), (3.8). The mapping $\phi_{0}$ equally satisfies (3.6) and (3.9).

We set

$$
\tau_{0}: \boldsymbol{V} \rightarrow \mathbb{R}, \quad \tau_{0}(\boldsymbol{v})=\left|v_{N}^{1}+v_{N}^{2}\right|_{\Xi}+|\boldsymbol{v}| \quad \forall \boldsymbol{v} \in \boldsymbol{V}
$$

The mapping $\tau_{0}$, which clearly verifies (3.10), is weakly continuous on $\boldsymbol{V}$, since it contains a trace term considered in $L^{2}(\Xi)$ and the norm in $\boldsymbol{H}$.

As the function $s \mapsto(s-g)_{+}$is Lipschitz continuous on $\mathbb{R}$, using (3.10) we also have

$$
\begin{aligned}
& \exists \tilde{\eta}_{\varepsilon}, \eta_{\varepsilon}>0 \quad\left|p_{\varepsilon}\left(\boldsymbol{u}_{1}, \boldsymbol{w}_{1}\right)-p_{\varepsilon}\left(\boldsymbol{u}_{1}, \boldsymbol{w}_{2}\right)+p_{\varepsilon}\left(\boldsymbol{u}_{2}, \boldsymbol{w}_{2}\right)-p_{\varepsilon}\left(\boldsymbol{u}_{2}, \boldsymbol{w}_{1}\right)\right| \\
& =\left|p_{\varepsilon}\left(\boldsymbol{u}_{1}, \boldsymbol{w}_{1}-\boldsymbol{w}_{2}\right)-p_{\varepsilon}\left(\boldsymbol{u}_{2}, \boldsymbol{w}_{1}-\boldsymbol{w}_{2}\right)\right| \\
& \leq \tilde{\eta}_{\varepsilon}\left|\tau_{0}\left(\boldsymbol{u}_{1}-\boldsymbol{u}_{2}\right)\right|\left\|\boldsymbol{w}_{1}-\boldsymbol{w}_{2}\right\| \leq \eta_{\varepsilon}\left\|\boldsymbol{u}_{1}-\boldsymbol{u}_{2}\right\|\left\|\boldsymbol{w}_{1}-\boldsymbol{w}_{2}\right\| \\
& \quad \forall \boldsymbol{u}_{1,2}, \boldsymbol{w}_{1,2} \in \boldsymbol{V},
\end{aligned}
$$


and

$$
\begin{aligned}
\exists \tilde{\eta}_{c}, & \eta_{c}>0\left|c\left(\lambda_{1}, \boldsymbol{u}_{1}, \boldsymbol{w}_{1}\right)-c\left(\lambda_{1}, \boldsymbol{u}_{1}, \boldsymbol{w}_{2}\right)+c\left(\lambda_{2}, \boldsymbol{u}_{2}, \boldsymbol{w}_{2}\right)-c\left(\lambda_{2}, \boldsymbol{u}_{2}, \boldsymbol{w}_{1}\right)\right| \\
= & \left|c\left(\lambda_{1}, \boldsymbol{u}_{1}, \boldsymbol{w}_{1}-\boldsymbol{w}_{2}\right)-c\left(\lambda_{2}, \boldsymbol{u}_{2}, \boldsymbol{w}_{1}-\boldsymbol{w}_{2}\right)\right| \\
\leq & \tilde{\eta}_{c}\left(\left|\lambda_{1}-\lambda_{2}\right|_{\Xi}+\left|\tau_{0}\left(\boldsymbol{u}_{1}-\boldsymbol{u}_{2}\right)\right|\right)\left\|\boldsymbol{w}_{1}-\boldsymbol{w}_{2}\right\| \\
\leq & \eta_{c}\left(\left|\lambda_{1}-\lambda_{2}\right|_{\Xi}+\left\|\boldsymbol{u}_{1}-\boldsymbol{u}_{2}\right\|\right)\left\|\boldsymbol{w}_{1}-\boldsymbol{w}_{2}\right\| \\
& \forall \lambda_{1,2} \in \Lambda, \boldsymbol{u}_{1,2}, \boldsymbol{w}_{1,2} \in \boldsymbol{V}
\end{aligned}
$$

Using the relations (2.11) and (3.10) one can clearly establish the following estimates: $\exists \tilde{\eta}_{J}, \eta_{J}>0$ such that

$$
\begin{aligned}
& \left|J\left(\lambda_{1}, \boldsymbol{u}_{1}, \boldsymbol{v}_{1}, \boldsymbol{w}_{1}\right)-J\left(\lambda_{1}, \boldsymbol{u}_{1}, \boldsymbol{v}_{1}, \boldsymbol{w}_{2}\right)+J\left(\lambda_{2}, \boldsymbol{u}_{2}, \boldsymbol{v}_{2}, \boldsymbol{w}_{2}\right)-J\left(\lambda_{2}, \boldsymbol{u}_{2}, \boldsymbol{v}_{2}, \boldsymbol{w}_{1}\right)\right| \\
& \leq \tilde{\eta}_{J}\left(\left|\lambda_{1}-\lambda_{2}\right|_{\Xi}+\left|\tau_{0}\left(\boldsymbol{u}_{1}-\boldsymbol{u}_{2}\right)\right|+\left|\boldsymbol{v}_{1}-\boldsymbol{v}_{2}\right|\right) \| \boldsymbol{w}_{1}-\boldsymbol{w}_{2}|| \\
& \leq \eta_{J}\left(\left|\lambda_{1}-\lambda_{2}\right|_{\Xi}+\| \boldsymbol{u}_{1}-\boldsymbol{u}_{2}|+| \boldsymbol{v}_{1}-\boldsymbol{v}_{2} \mid\right)\left\|\boldsymbol{w}_{1}-\boldsymbol{w}_{2}\right\| \\
& \quad \forall \lambda_{1,2} \in \Lambda, \boldsymbol{u}_{1,2}, \boldsymbol{v}_{1,2}, \boldsymbol{w}_{1,2} \in \boldsymbol{V}
\end{aligned}
$$

From (4.2)-(4.4) it follows that (3.12) is satisfied and if we set $\boldsymbol{w}=\boldsymbol{w}_{1}$, $\boldsymbol{w}_{2}=\mathbf{0}$, we obtain (3.11). Finally, relation (2.12) implies the validity of (3.13).

Thus, by Theorem 3.5 the problem $\mathcal{P}_{\varepsilon}$ admits a unique solution.

\subsection{Existence of a variational solution}

We shall use several times the following compactness theorem proved by J. Simon [27].

Theorem 4.2. Let $X, U$ and $Y$ be three Banach spaces such that $X \subset U \subset Y$ with compact imbedding $X \rightarrow U$.

Let $\mathcal{F}$ be bounded in $L^{p}(0, T ; X)$, where $1 \leq p<\infty$, and $\partial \mathcal{F} / \partial t=\{\dot{f} ; f \in$ $\mathcal{F}\}$ be bounded in $L^{1}(0, T ; Y)$. Then $\mathcal{F}$ is relatively compact in $L^{p}(0, T ; U)$.

Let $\mathcal{F}$ be bounded in $L^{\infty}(0, T ; X)$ and $\partial \mathcal{F} / \partial t$ be bounded in $L^{r}(0, T ; Y)$, where $r>1$. Then $\mathcal{F}$ is relatively compact in $C^{0}([0, T] ; U)$.

Theorem 4.3. Under the assumptions of Section 2 there exists a solution of the problem $\mathcal{P}_{v}$.

Proof. First, we establish some estimates on the penalized solutions $\boldsymbol{u}_{\varepsilon}$ and $\beta_{\varepsilon}$ which will enable us to pass to the limit in $\mathcal{P}_{\varepsilon}$ in order to obtain a solution 
of $\mathcal{P}_{v}$. If we choose $\boldsymbol{w}=\mathbf{0}$ in (2.16), by integrating from 0 to $\left.t \in\right] 0, T$ [ we have

$$
\begin{aligned}
& \int_{0}^{t}\left(\ddot{\boldsymbol{u}}_{\varepsilon}, \dot{\boldsymbol{u}}_{\varepsilon}\right) d s+\int_{0}^{t} a\left(\boldsymbol{u}_{\varepsilon}, \dot{\boldsymbol{u}}_{\varepsilon}\right) d s+\int_{0}^{t} b\left(\dot{\boldsymbol{u}}_{\varepsilon}, \dot{\boldsymbol{u}}_{\varepsilon}\right) d s \\
& \quad+\int_{0}^{t} p_{\varepsilon}\left(\boldsymbol{u}_{\varepsilon}, \dot{\boldsymbol{u}}_{\varepsilon}\right) d s+\int_{0}^{t} c\left(\beta_{\varepsilon}, \boldsymbol{u}_{\varepsilon}, \dot{\boldsymbol{u}}_{\varepsilon}\right) d s \leq \int_{0}^{t}\left\langle\boldsymbol{L}, \dot{\boldsymbol{u}}_{\varepsilon}\right\rangle d s
\end{aligned}
$$

As $a$ is a symmetric bilinear mapping, $g_{0}$ is independent of time and $\boldsymbol{u}_{0}$ belongs to $\boldsymbol{K}$, we obtain for all $t \in] 0, T[$

$$
\begin{array}{r}
\frac{1}{2}\left|\dot{\boldsymbol{u}}_{\varepsilon}(t)\right|^{2}+\frac{1}{2} a\left(\boldsymbol{u}_{\varepsilon}(t), \boldsymbol{u}_{\varepsilon}(t)\right)+\int_{0}^{t} b\left(\dot{\boldsymbol{u}}_{\varepsilon}, \dot{\boldsymbol{u}}_{\varepsilon}\right) d s+\frac{1}{2 \varepsilon}\left|\vartheta\left(\left[u_{\varepsilon N}(t)\right]_{+}\right)\right|_{\Xi}^{2} \\
\leq \int_{0}^{t}\left\langle\boldsymbol{L}, \dot{\boldsymbol{u}}_{\varepsilon}\right\rangle d s-\int_{0}^{t} c\left(\beta_{\varepsilon}, \boldsymbol{u}_{\varepsilon}, \dot{\boldsymbol{u}}_{\varepsilon}\right) d s+\frac{1}{2}\left|\boldsymbol{u}_{1}\right|^{2}+\frac{1}{2} a\left(\boldsymbol{u}_{0}, \boldsymbol{u}_{0}\right) .
\end{array}
$$

Using (4.1), Young's inequality, the properties of the truncation operator $\vartheta$ and Gronwall's lemma, it follows that there exists a positive constant $M$ independent of $\varepsilon$ such that, for all $\varepsilon>0$, the following estimates on $\boldsymbol{u}_{\varepsilon}$ hold

$$
\begin{aligned}
& \left.\left|\dot{\boldsymbol{u}}_{\varepsilon}(t)\right| \leq M,\left\|\boldsymbol{u}_{\varepsilon}(t)\right\| \leq M \quad\left|\left[u_{\varepsilon N}(t)\right]_{+}\right|_{\Xi} \leq M \sqrt{\varepsilon} \quad \forall t \in\right] 0, T[, \\
& \int_{0}^{T}\left\|\dot{\boldsymbol{u}}_{\varepsilon}\right\|^{2} d s \leq M .
\end{aligned}
$$

From (2.16) we obtain for all $\boldsymbol{\varphi}=\left(\boldsymbol{\varphi}^{\mathbf{1}}, \boldsymbol{\varphi}^{\mathbf{2}}\right) \in L^{2}\left(0, T ;\left[H_{0}^{1}\left(\Omega^{1}\right)\right]^{d} \times\left[H_{0}^{1}\left(\Omega^{2}\right)\right]^{d}\right)$

$$
\int_{0}^{T}\left(\ddot{\boldsymbol{u}}_{\varepsilon}, \boldsymbol{\varphi}\right) d t+\int_{0}^{T} a\left(\boldsymbol{u}_{\varepsilon}, \boldsymbol{\varphi}\right) d t+\int_{0}^{T} b\left(\dot{\boldsymbol{u}}_{\varepsilon}, \boldsymbol{\varphi}\right) d t=\sum_{\alpha=1,2} \int_{0}^{T} \int_{\Omega^{\alpha}} \boldsymbol{f}^{\alpha} \cdot \boldsymbol{\varphi}^{\alpha} d x d t
$$

Hence, the term $\ddot{\boldsymbol{u}}_{\varepsilon}$ is bounded in $L^{2}\left(0, T ; \boldsymbol{H}^{-1}\right)$ by a constant independent of $\varepsilon$.

For all $\boldsymbol{v} \in L^{\infty}(0, T ; \boldsymbol{V}) \cap W^{1,2}(0, T ; \boldsymbol{H})$ such that $\boldsymbol{v}(t) \in \boldsymbol{K}$ for almost every $t \in] 0, T$, we choose in (2.16) $\boldsymbol{w}=\dot{\boldsymbol{u}}_{\varepsilon}+\frac{1}{\kappa}\left(\boldsymbol{v}-\boldsymbol{u}_{\varepsilon}\right)$. Then, integrating with respect to $t \in] 0, T$ [ from (2.16), we obtain

$$
\begin{aligned}
& \int_{0}^{T}\left(\ddot{\boldsymbol{u}}_{\varepsilon}, \boldsymbol{v}-\boldsymbol{u}_{\varepsilon}\right) d t+\int_{0}^{T} a\left(\boldsymbol{u}_{\varepsilon}, \boldsymbol{v}-\boldsymbol{u}_{\varepsilon}\right) d t+\int_{0}^{T} b\left(\dot{\boldsymbol{u}}_{\varepsilon}, \boldsymbol{v}-\boldsymbol{u}_{\varepsilon}\right) d t \\
& \quad+\int_{0}^{T} p_{\varepsilon}\left(\boldsymbol{u}_{\varepsilon}, \boldsymbol{v}-\boldsymbol{u}_{\varepsilon}\right) d t+\int_{0}^{T} c\left(\beta_{\varepsilon}, \boldsymbol{u}_{\varepsilon}, \boldsymbol{v}-\boldsymbol{u}_{\varepsilon}\right) d t \\
& +\int_{0}^{T}\left\{J\left(\beta_{\varepsilon}, \boldsymbol{u}_{\varepsilon}, \dot{\boldsymbol{u}}_{\varepsilon}, \boldsymbol{v}+\kappa \dot{\boldsymbol{u}}_{\varepsilon}-\boldsymbol{u}_{\varepsilon}\right)-J\left(\beta_{\varepsilon}, \boldsymbol{u}_{\varepsilon}, \dot{\boldsymbol{u}}_{\varepsilon}, \kappa \dot{\boldsymbol{u}}_{\varepsilon}\right)\right\} d t \geq \int_{0}^{T}\left\langle\boldsymbol{L}, \boldsymbol{v}-\boldsymbol{u}_{\varepsilon}\right\rangle d t .
\end{aligned}
$$


Integrating by parts the acceleration term in (4.6) and by a monotonicity argument for $p_{\varepsilon}$ we derive

$$
\begin{aligned}
& \left\langle\dot{\boldsymbol{u}}_{\varepsilon}(T), \boldsymbol{v}(T)-\boldsymbol{u}_{\varepsilon}(T)\right\rangle_{-1 / 2,1 / 2}-\left(\boldsymbol{u}_{1}, \boldsymbol{v}(0)-\boldsymbol{u}_{0}\right)-\int_{0}^{T}\left(\dot{\boldsymbol{u}}_{\varepsilon}, \dot{\boldsymbol{v}}-\dot{\boldsymbol{u}}_{\varepsilon}\right) d t \\
& \quad+\int_{0}^{T}\left\{a\left(\boldsymbol{u}_{\varepsilon}, \boldsymbol{v}-\boldsymbol{u}_{\varepsilon}\right)+b\left(\dot{\boldsymbol{u}}_{\varepsilon}, \boldsymbol{v}-\boldsymbol{u}_{\varepsilon}\right)+c\left(\beta_{\varepsilon}, \boldsymbol{u}_{\varepsilon}, \boldsymbol{v}-\boldsymbol{u}_{\varepsilon}\right)\right\} d t \\
& +\int_{0}^{T}\left\{J\left(\beta_{\varepsilon}, \boldsymbol{u}_{\varepsilon}, \dot{\boldsymbol{u}}_{\varepsilon}, \boldsymbol{v}+\kappa \dot{\boldsymbol{u}}_{\varepsilon}-\boldsymbol{u}_{\varepsilon}\right)-J\left(\beta_{\varepsilon}, \boldsymbol{u}_{\varepsilon}, \dot{\boldsymbol{u}}_{\varepsilon}, \kappa \dot{\boldsymbol{u}}_{\varepsilon}\right)\right\} d t \geq \int_{0}^{T}\left\langle\boldsymbol{L}, \boldsymbol{v}-\boldsymbol{u}_{\varepsilon}\right\rangle d t \\
& \left.\quad \forall \boldsymbol{v} \in L^{\infty}(0, T ; \boldsymbol{V}) \cap W^{1,2}(0, T ; \boldsymbol{H}) \text { with } \boldsymbol{v}(t) \in \boldsymbol{K} \text { a.e. } t \in\right] 0, T[.
\end{aligned}
$$

From the estimates (4.5) and the previous estimate on the acceleration, it follows that there exists $\boldsymbol{u}$ such that, up to a subsequence,

$$
\begin{array}{ll}
\boldsymbol{u}_{\varepsilon} \rightarrow^{*} \boldsymbol{u} \text { in } L^{\infty}(0, T ; \boldsymbol{V}), & \dot{\boldsymbol{u}}_{\varepsilon} \rightarrow \dot{\boldsymbol{u}} \text { in } L^{2}(0, T ; \boldsymbol{V}), \\
\dot{\boldsymbol{u}}_{\varepsilon} \boldsymbol{\rightarrow}^{*} \dot{\boldsymbol{u}} \text { in } L^{\infty}(0, T ; \boldsymbol{H}), \quad \ddot{\boldsymbol{u}}_{\varepsilon} \rightarrow \ddot{\boldsymbol{u}} \text { in } L^{2}\left(0, T ; \boldsymbol{H}^{-1}\right) .
\end{array}
$$

As $W^{1,2}(0, T ; \boldsymbol{V}) \subset C^{0}([0, T] ; \boldsymbol{V})$, it follows that, for all $t \in[0, T],\left(\boldsymbol{u}_{\varepsilon}(t)\right)_{\varepsilon}$ is bounded in $\boldsymbol{V}$ by a constant independent of $\varepsilon$ and of $t$, so that by a diagonal process we can extract a subsequence, still denoted by $\left(\boldsymbol{u}_{\varepsilon}\right)_{\varepsilon}$, such that

$$
\boldsymbol{u}_{\varepsilon}(t) \rightarrow \boldsymbol{u}(t) \text { in } \boldsymbol{V} \quad \forall t \in[0, T] .
$$

By (4.8) we can easily pass to the limit in the linear terms of (4.7). To pass to the limit in the nonlinear terms we need the compactness result of Theorem 4.2. As $\partial \Omega^{\alpha}$ is Lipschitz continuous, the imbeddings from $\boldsymbol{V}^{\alpha}$ into $\boldsymbol{H}^{\alpha}$, from $\boldsymbol{V}^{\alpha}$ into $\left[H^{1 / 2}\left(\Omega^{\alpha}\right)\right]^{d}$ and from $\boldsymbol{H}^{\alpha}$ into $\left[H^{-1 / 2}\left(\Omega^{\alpha}\right)\right]^{d}$ are compact, $\alpha=1,2$. Then we may apply Theorem 4.2 with

$$
\begin{array}{ll}
\mathcal{F}=\left(\dot{\boldsymbol{u}}_{\varepsilon}\right)_{\varepsilon}, & X=\boldsymbol{V}, U=\boldsymbol{H}, Y=\boldsymbol{H}^{-1}, p=2, \\
\mathcal{F}=\left(\boldsymbol{u}_{\varepsilon}\right)_{\varepsilon}, & X=\boldsymbol{V}, U=\boldsymbol{H}^{1 / 2}, Y=\boldsymbol{H}, r=2, \\
\mathcal{F}=\left(\dot{\boldsymbol{u}}_{\varepsilon}\right)_{\varepsilon}, & X=\boldsymbol{H}, U=\boldsymbol{H}^{-1 / 2}, Y=\boldsymbol{H}^{-1}, r=2,
\end{array}
$$

so that, up to a subsequence, we obtain

$$
\begin{aligned}
& \dot{\boldsymbol{u}}_{\varepsilon} \rightarrow \dot{\boldsymbol{u}} \text { in } L^{2}(0, T ; \boldsymbol{H}), \quad \boldsymbol{u}_{\varepsilon} \rightarrow \boldsymbol{u} \text { in } C^{0}\left([0, T] ; \boldsymbol{H}^{1 / 2}\right), \\
& \dot{\boldsymbol{u}}_{\varepsilon} \rightarrow \dot{\boldsymbol{u}} \text { in } C^{0}\left([0, T] ; \boldsymbol{H}^{-1 / 2}\right) .
\end{aligned}
$$

Hence,

$$
\left\langle\dot{\boldsymbol{u}}_{\varepsilon}(T), \boldsymbol{v}(T)-\boldsymbol{u}_{\varepsilon}(T)\right\rangle_{-1 / 2,1 / 2} \rightarrow\langle\dot{\boldsymbol{u}}(T), \boldsymbol{v}(T)-\boldsymbol{u}(T)\rangle_{-1 / 2,1 / 2} .
$$


The functional $\boldsymbol{v} \mapsto \int_{0}^{T} a(\boldsymbol{v}, \boldsymbol{v}) d t$ is convex and continuous on $L^{2}(0, T ; \boldsymbol{V})$, so it is sequentially weakly lower semicontinuous, which implies that

$$
\liminf _{\varepsilon \rightarrow 0} \int_{0}^{T} a\left(\boldsymbol{u}_{\varepsilon}, \boldsymbol{u}_{\varepsilon}\right) d t \geq \int_{0}^{T} a(\boldsymbol{u}, \boldsymbol{u}) d t
$$

Since $\boldsymbol{v} \mapsto b(\boldsymbol{v}, \boldsymbol{v})$ is convex and continuous on $\boldsymbol{V}$, it is sequentially weakly lower semicontinuous on $\boldsymbol{V}$. Thus, as $\boldsymbol{u}_{\varepsilon}(T) \rightarrow \boldsymbol{u}(T)$ in $\boldsymbol{V}$, we obtain

$$
\begin{aligned}
& \liminf _{\varepsilon \rightarrow 0} \int_{0}^{T} b\left(\dot{\boldsymbol{u}}_{\varepsilon}, \boldsymbol{u}_{\varepsilon}\right) d t=\liminf _{\varepsilon \rightarrow 0}\left(\frac{1}{2} b\left(\boldsymbol{u}_{\varepsilon}(T), \boldsymbol{u}_{\varepsilon}(T)\right)-\frac{1}{2} b\left(\boldsymbol{u}_{0}, \boldsymbol{u}_{0}\right)\right) \\
& \geq \frac{1}{2} b(\boldsymbol{u}(T), \boldsymbol{u}(T))-\frac{1}{2} b\left(\boldsymbol{u}_{0}, \boldsymbol{u}_{0}\right)=\int_{0}^{T} b(\dot{\boldsymbol{u}}, \boldsymbol{u}) d t .
\end{aligned}
$$

As the imbedding from $H^{1 / 2}(\Xi)$ into $L^{2}(\Xi)$ is compact, by (4.9) we have

$$
\left[u_{\varepsilon N}\right](t) \rightarrow\left[u_{N}\right](t) \text { in } L^{2}(\Xi) \quad \forall t \in[0, T] .
$$

Then from $(4.5)_{3}$ we derive

$$
0=\lim _{\varepsilon \rightarrow 0}\left|\left[u_{\varepsilon N}(t)\right]_{+}\right|_{\Xi}=\left|\left[u_{N}\right]_{+}(t)\right|_{\Xi} \quad \forall t \in[0, T] .
$$

Hence, $\left[u_{N}(t)\right] \leq 0$ almost everywhere on $\Xi$ and for all $t \in[0, T]$, which implies $\boldsymbol{u}(t) \in \boldsymbol{K}$ for all $t \in[0, T]$.

Let $\beta:=\beta_{\boldsymbol{u}} \in W^{1, \infty}\left(0, T ; L^{\infty}(\Xi)\right)$, with $\beta(s) \in \Lambda$ for all $\left.s \in\right] 0, T[$, be the solution, which by Proposition 3.3 exists and is unique, of the parabolic variational inequality

$$
(\dot{\beta}, \lambda-\beta)_{\Xi}+\gamma(\boldsymbol{u}, \beta, \lambda-\beta) \geq(\chi, \lambda-\beta)_{\Xi} \quad \forall \lambda \in \Lambda
$$

with the initial condition $\beta_{0}$.

We will verify that $(\boldsymbol{u}, \beta)$ is a solution of the problem $\mathcal{P}_{v}$. By a similar result as in Lemma 3.4, it follows that there exists a constant $M^{\prime}>0$, independent of $\boldsymbol{u}_{\varepsilon}, \boldsymbol{u}, \beta_{\varepsilon}, \beta$, such that for all $t \in[0, T]$

$$
\left|\beta_{\varepsilon}(t)-\beta(t)\right|_{\Xi}^{2} \leq M^{\prime} \int_{0}^{t}\left|\left[u_{\varepsilon N}\right](s)-\left[u_{N}\right](s)\right|_{\Xi}^{2} d s .
$$

Then by (4.5) 2 and (4.14) we have

$$
\beta_{\varepsilon}(t) \rightarrow \beta(t) \text { in } L^{2}(\Xi) \quad \forall t \in[0, T] .
$$

To pass to the limit in the friction term, we apply Theorem 4.2 with $\mathcal{F}=$ $\left(\dot{\boldsymbol{u}}_{\varepsilon}\right)_{\varepsilon}, X=\boldsymbol{V}, U=\boldsymbol{H}^{1-\iota}$, where $1 / 2>\iota>0, Y=\boldsymbol{H}^{-1}, p=2$ and we obtain

$$
\boldsymbol{u}_{\varepsilon} \rightarrow \boldsymbol{u} \text { in } W^{1,2}\left(0, T ; \boldsymbol{H}^{1-\iota}\right) .
$$


As the mapping $\boldsymbol{v} \mapsto\left[\boldsymbol{v}_{T}\right]$ defined from $\boldsymbol{H}^{1-\iota}$ into $\left[L^{2}(\Xi)\right]^{d}$ is compact, we have

$$
\left[\boldsymbol{u}_{\varepsilon T}\right] \rightarrow\left[\boldsymbol{u}_{T}\right] \text { in } W^{1,2}\left(0, T ;\left[L^{2}(\Xi)\right]^{d}\right) .
$$

Relations (2.11), (4.11)-(4.14), (4.17) and (4.18) enable us to pass to the lower limit in (4.7) and to prove that $(\boldsymbol{u}, \beta)$ verify (2.13).

Now, integrating with respect to $t \in] 0, T[$ from (2.17) we derive

$$
\begin{gathered}
\int_{0}^{T}\left(\dot{\beta}_{\varepsilon}, \lambda-\beta_{\varepsilon}\right)_{\Xi} d t+\int_{0}^{T} \gamma\left(\boldsymbol{u}_{\varepsilon}, \beta_{\varepsilon}, \lambda-\beta_{\varepsilon}\right) d t \geq \int_{0}^{T}\left(\chi, \lambda-\beta_{\varepsilon}\right)_{\Xi} d t \\
\left.\forall \lambda \in L^{2}\left(0, T ; L^{2}(\Xi)\right) \text { with } \lambda(t) \in \Lambda \text { a.e. } t \in\right] 0, T[
\end{gathered}
$$

so that using (4.14), (4.17) and a weakly lower semicontinuity argument, by passing to the lower limit we obtain

$$
\begin{gathered}
\int_{0}^{T}(\dot{\beta}, \lambda-\beta)_{\Xi} d t+\int_{0}^{T} \gamma(\boldsymbol{u}, \beta, \lambda-\beta) d t \geq \int_{0}^{T}(\chi, \lambda-\beta)_{\Xi} d t \\
\left.\forall \lambda \in L^{2}\left(0, T ; L^{2}(\Xi)\right) \text { with } \lambda(t) \in \Lambda \text { a.e. } t \in\right] 0, T[.
\end{gathered}
$$

By Lebesgue's theorem, it follows that $(\boldsymbol{u}, \beta)$ verify also the parabolic inequality (2.14).

We note that the abstract results presented in section 3 can be also applied to prove the existence and uniqueness of solutions to dynamic contact problems with normal compliance and adhesion between a viscoelastic body and an obstacle.

Similar results as the previous ones are easily seen to hold for the contact with irreversible adhesion, where the evolution of the intensity of adhesion is governed by a differential equation satisfying the relation (3.27), see, e.g., [28].

\section{$5 \quad$ Numerical examples}

In this section, an example is presented to illustrate the mechanical behaviour associated with this interface law and to study the influence of the main mechanical parameters on the solution. The dynamic behaviour of a cylindrical viscoelastic block in adhesive frictional contact $(\mu=0.2)$ under axisymmetric conditions with a stiff sphere is analysed, see Figure 1.

The previous sections concern a dynamic contact problem in viscoelasticity, which constitutes an extension of the model considered in [22, 24] to the case of recoverable adhesion. In elastodynamics, the discrete problem, 
the algorithms and the numerical solution were given in [24, 1] for non recoverable adhesion. They are based on a combination of the Non Smooth Contact Dynamics (NSCD) method, developed by M. Jean and J.J. Moreau [15, 21, 11], and the methods proposed in [22, 24] for the treatment of the adhesion. This approach is extended here to viscoelasticity and to healing process, see also [25, 26].
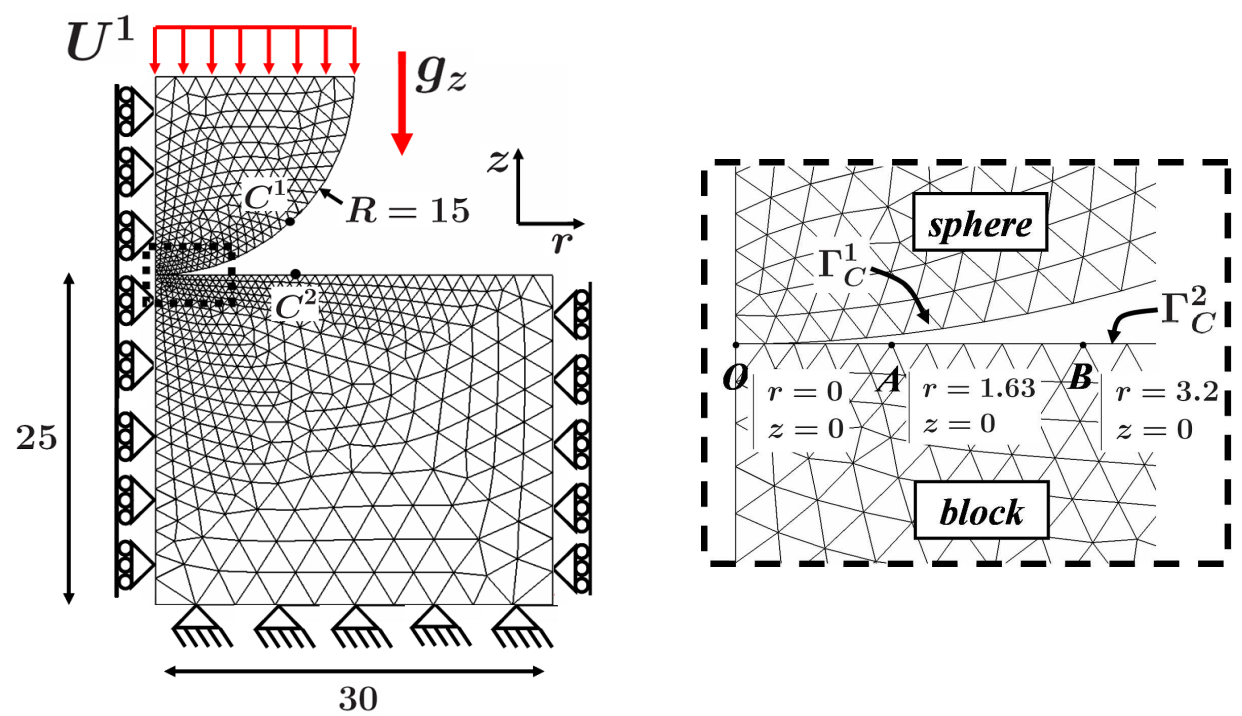

Figure 1: Initial configurations, boundary conditions, finite element discretization of the problems and points $O(r=0, z=0), A(1.63,0), B(3.2,0)$. $\Gamma_{C}^{1}$ and $\Gamma_{C}^{2}$ are bounded by $O, C^{1}(10.1,3.98)$ and $O, C^{2}(9.82,0)$, respectively (length unit is $\mathrm{mm}$ ).

The evolution of the adhesion $\beta$ is computed by using an implicit method and so a fixed point on $\beta$ is necessary (the convergence test on the relative variation is taken as $10^{-5}$ ). The convergence of the relaxation method included in the NSCD algorithm is tested on the relative variation of the contact power (test condition : $10^{-5}$ ). The implementation of the model with recoverable adhesion was conducted in the finite element code LMGC90 [11].

The finite element discretizations consist of $P_{1}$ elements and an initial triangulation with 480 nodes for the cross-section of the block and 270 nodes for the cross-section of the sphere is considered. The corresponding size of minimal length of edge mesh is $h_{c}=0.33 \mu \mathrm{m}$. A $\theta$-method is used to solve the dynamic equations and the choice $\theta=0.51$ gives good stability and a small numerical damping.

The sphere is initially in contact with the viscoelastic block at the point $O$. A compressive cycle is imposed with a prescribed displacement $\boldsymbol{U}^{1}(t)=$ 
$u_{z}(t) \boldsymbol{e}_{z}$ on the upper part of the sphere, see Figure 2, The viscoelastic con-

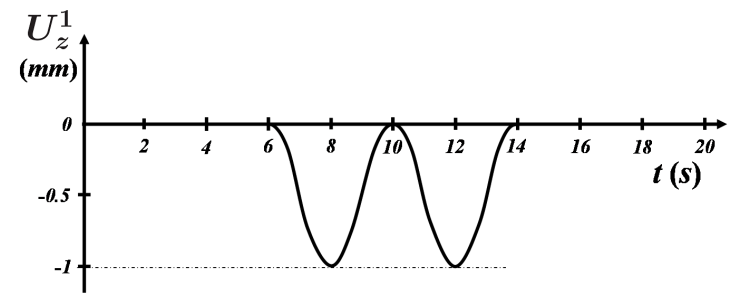

Figure 2: Imposed displacement on the upper face of the sphere $u_{z}$.

stitutive law is described by the following relations:

$$
\begin{aligned}
& \boldsymbol{\sigma}=\boldsymbol{\sigma}^{r}+\boldsymbol{\sigma}^{i r}, \\
& \boldsymbol{\varepsilon}=\frac{1+\nu}{E} \boldsymbol{\sigma}^{r}-\frac{\nu}{E} \operatorname{tr}\left(\boldsymbol{\sigma}^{r}\right) \boldsymbol{I}, \\
& \dot{\boldsymbol{\varepsilon}}=\frac{1+\tilde{\nu}}{\tilde{E}} \boldsymbol{\sigma}^{i r}-\frac{\tilde{\nu}}{\tilde{E}} \operatorname{tr}\left(\boldsymbol{\sigma}^{i r}\right) \boldsymbol{I},
\end{aligned}
$$

where $E$ is Young's modulus, $\nu$ is Poisson's ratio and $\tilde{E}, \tilde{\nu}$ are the viscosity coefficients. We considered for the sphere $E^{(1)}=2.10^{11} \mathrm{~Pa}, \nu^{(1)}=0.3$, $\rho^{(1)}=7800 \mathrm{~kg} \cdot \mathrm{m}^{-3}, \tilde{E}^{(1)}=\eta^{(1)} E^{(1)}$ with $\eta^{(1)} \rightarrow 0$, and for the block $\tilde{E}^{(2)}=$ $\eta^{(2)} E^{(2)}$ with $\left.\left.\eta:=\eta^{(2)} \in\right] 0,10\right] \mathrm{s}, E^{(2)}=5.10^{6} \mathrm{~Pa}, \nu^{(2)}=\tilde{\nu}^{(2)}=0.48$, $\rho^{(2)}=1000 \mathrm{~kg} \cdot \mathrm{m}^{-3}$.

This example is used to investigate the dependency of the solution on the variation of the following parameters:

- the adhesion parameters $C_{N}$ and $w$,

- the volumic viscosity parameter $\eta$,

- the mesh size.

\subsection{Effect of adhesion parameters}

We consider here three cases of interfacial behaviour with different choices of the adhesion parameters $C_{N}$ and $w$ as given in Table 1 .

For $\eta=1 \mathrm{~s}$, the evolution of $\beta$ at the candidate contact point $B$ (see Figure 11) and the evolution of contact radius, characterized by the relation $g=0$, in these three cases are presented in Figures 3 and 4 , respectively.

In case 1 , the bonds are weak and brittle. Adhesion has minor effect on the solution and a loss of contact is observed. In case 2, the bonds are strong and brittle. An initial contact is observed (radius $0.9 \mathrm{~mm}$ ), which is due to the stronger adhesion that acts during the initial step, when only gravity is 


\begin{tabular}{lcccc}
\hline & Bonds behaviour & $w\left({\left.\mathrm{~J} . \mathrm{m}^{-2}\right)}\right.$ & $C_{N}\left({\left.\mathrm{~N} . \mathrm{m}^{-3}\right)}^{-}\right.$ & $b\left(\mathrm{~N} . \mathrm{s} . \mathrm{m}^{-1}\right)$ \\
\hline case 1 & weak and brittle & 1 & $10^{8}$ & 0.1 \\
case 2 & strong and brittle & 30 & $3 \times 10^{9}$ & 0.1 \\
case 3 & strong and resilient & 100 & $10^{10}$ & 0.1 \\
\hline
\end{tabular}

Table 1: Three cases of interfacial behaviour

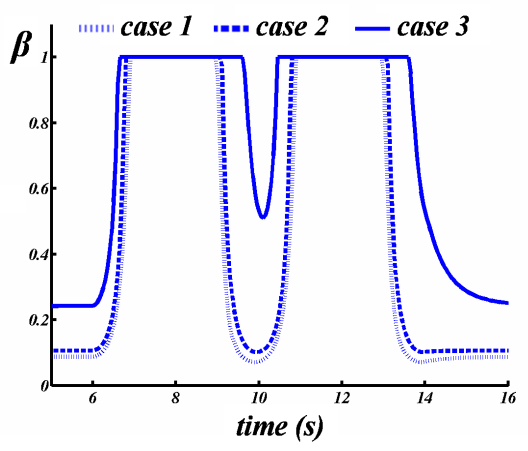

Figure 3: Evolution of $\beta$ at point $B$ in three cases.

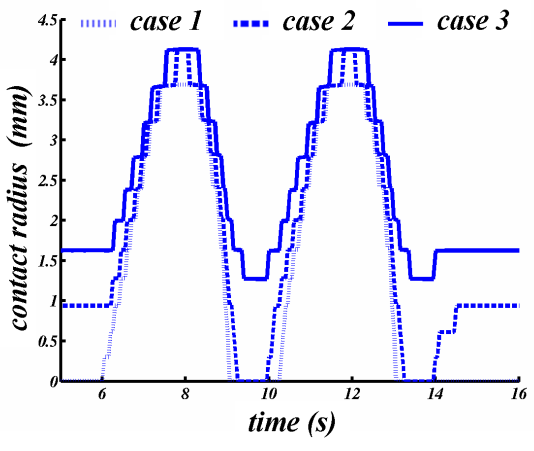

Figure 4: Evolution of the contact radius $(g=0)$ in three cases.

concerned. A loss of contact is still observed at $t=10 \mathrm{~s}$ because the evolution of the adhesion is still brittle. In case 3, the bonds are strong and resilient. Adhesion has major effect on the solution and the two bodies remain always in contact even at $t=10 \mathrm{~s}$.

\subsection{Effect of the volumic viscosity}

The evolutions of the displacement vector and of the intensity of adhesion in case 1, as presented in Figures 5 and [6, show a good convergence of viscoelastic solutions towards an elastic solution as $\eta$ tends to 0 .

\subsection{Effect of the mesh size}

We consider the case 3 with $\eta$ equal to $1 \mathrm{~s}$. The distributions of the normal and tangential components of the stress vector, $\sigma_{N}$ and $\sigma_{T}$, versus the position $r$ are displayed at $t=8 \mathrm{~s}$ in Figure 7 for different meshes, as given in Table 2,

The bodies are in contact $\left(\sigma_{N} \leq 0\right.$ and $\left.g=0\right)$ for $r \leq r_{C}$, where $r_{C} \simeq 4.5$ $\mathrm{mm}$ is the contact radius, and the bodies are in adhesion $\left(\sigma_{N} \geq 0\right)$ for 

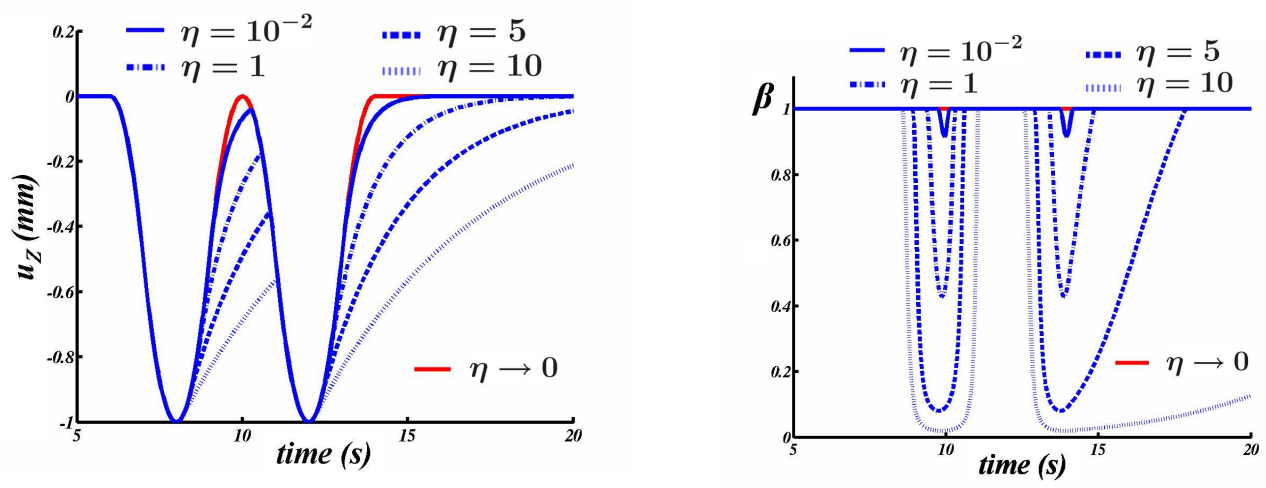

Figure 5: Evolutions of the displacement at point $O$ for different values of $\eta$ in case 1.

Figure 6: Evolutions of $\beta$ at point $A$ for different values of $\eta$ in case 1 .

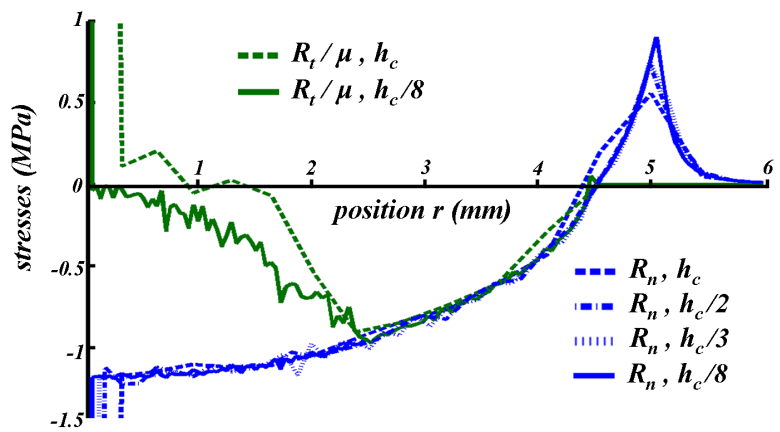

Figure 7: Distributions of the normal and tangential interfacial stress $\sigma_{N}$ and $\sigma_{T}$ versus position $r$ for different mesh sizes of the interaction surfaces $\Gamma_{C}^{1}$ and $\Gamma_{C}^{2}\left(h_{c}=0,33 \mathrm{~mm}\right)$ at $t=8 \mathrm{~s}$.

\begin{tabular}{ccc}
\hline Name & Number of DOF & Number of contact element for $\Gamma_{C}^{1}$ and $\Gamma_{C}^{2}$ \\
\hline$h_{c}$ & 1500 & 30 \\
$h_{c} / 2$ & 2956 & 60 \\
$h_{c} / 3$ & 4390 & 90 \\
$h_{c} / 8$ & 14660 & 240 \\
\hline
\end{tabular}

Table 2: The different meshes.

$r_{A}>r>r_{C}$, where $r_{A} \simeq 5.5 \mathrm{~mm}$ is the adhesion radius. For $r \geq r_{A}$ the stress vector is negligible. 


\section{References}

[1] V. Acary and Y. Monerie, Formulation dynamique d'un modèle de zone cohésive tridimensionnelle couplant endommagement et interface. Revue Européenne des Eléments Finis, 10 (2001), 489-503.

[2] V. Barbu, Semigroups and Differential Equations in Banach Spaces. Noordhoff International Publishing, Leyden, The Netherlands 1976.

[3] M. Barquins, Sliding friction of rubber and Schallamach waves - A review. Materials Science and Engineering 73 (1985), 45-63.

[4] P. Boieri, F. Gastaldi and D. Kinderlehrer, Existence, uniqueness, and regularity results for the two-body contact problem. Appl. Math. Optim. 15 (1987), 251-277.

[5] H. Brézis, Problèmes unilatéraux. J. Math. Pures et Appl. 51 (1972), $1-168$.

[6] O. Chau, W. Han and M. Sofonea, A dynamic frictional contact problem with normal damped response. Acta Applicandae Mathematicae 71 (2002), 159-178.

[7] M. Cocou, E. Pratt and M. Raous, Formulation and approximation of quasistatic frictional contact. International Journal of Engineering Science. 34 (1996), 783-798.

[8] M. Cocou and R. Rocca, Existence results for unilateral quasistatic contact problems with friction and adhesion. Math. Modelling and Num. Analysis 34 (2000), 981-1001.

[9] M. Cocou and G. Scarella, Analysis of a dynamic unilateral contact problem for a cracked viscoelastic body. Z. Angew. Math. Phys. 57 (2006), 523-546.

[10] M. Cocou, M. Schryve and M. Raous, A variational analysis of a contact interaction problem in viscoelasticity. Proceedings of 6th Congress of Romanian Mathematicians, Bucharest, Romania, June 28 - July 4, 2007, Volume 1, Editura Academiei Romane, Bucharest 2009, 501-509.

[11] F. Dubois and M. Jean, LMGC90 - Une plateforme de développement dédiée à la modélisation de problèmes d'interaction. Actes du 6ème Colloque National en Calcul des Structures CSMA-AFM-LMS-Giens, Volume 1, M. Potier-Ferry, M. Bonnet and A. Bignonnet (eds.), Ecole Polytechnique, Paris 2003, 111-118. 
[12] C. Eck, J. Jarušek and M. Krbec, Unilateral Contact Problems - Variational Methods and Existence Theorems. Chapman\&Hall/CRC, Boca Raton, 2005.

[13] M. Frémond, Adhérence des solides. Journal de Mécanique Théorique et Appliquée 6 (1987), 383-407.

[14] M. Frémond, Contact with adhesion. Nonsmooth Mechanics and Applications, CISM Courses and Lectures No. 302, J.J. Moreau and P.D. Panagiotopoulos (eds.), Springer-Verlag, Wien-New York 1988, 177-221.

[15] M. Jean, The non smooth contact dynamics method. Comput. Meth. Appl. Mech. Engrg. 177 (1999), 235-257.

[16] K.L. Johnson, Mechanics of adhesion. Tribology International 31 (1998), 413-418.

[17] K.L. Johnson, K. Kendall and A.D. Roberts, Surface energy and the contact of elastic solids. Proc. R. Soc. Lond. A. 324 (1971), 301-313.

[18] K.L. Kuttler, Dynamic friction contact problems for general normal and friction laws. Nonlinear Anal. TMA 28 (1997), 559-575.

[19] K.L. Kuttler and M. Shillor, Dynamic contact with Signorini's condition and slip rate depending friction. Electronic J. Differential Equations $\mathbf{8 3}$ (2004), 1-21.

[20] J.A.C Martins and J.T. Oden, Existence and uniqueness results for dynamic contact problems with nonlinear normal and friction interface laws. Nonlinear Anal. TMA 11 (1987), 407-428.

[21] J.J. Moreau, Unilateral contact and dry friction in finite freedom dynamics. Nonsmooth Mechanics and Applications, CISM Courses and Lectures No. 302, J.J. Moreau and P.D. Panagiotopoulos (eds.), Springer-Verlag, Wien-New York 1988, 1-82.

[22] M. Raous, L. Cangémi and M. Cocou, A consistent model coupling adhesion, friction, and unilateral contact. Comput. Meth. Appl. Mech. Engrg. 177 (1999), 383-399.

[23] M. Raous and M.A. Karray, Model coupling friction and adhesion for steel-concrete interfaces. International Journal of Computer Application in Technology, 34 (2009), 42-50. 
[24] M. Raous and Y. Monerie, Unilateral contact, friction and adhesion: $3 D$ cracks in composite materials. Contact Mechanics, Proceedings of the 3rd Contact Mechanics International Symposium, Peniche, Portugal, June 17-21, 2001, J.A.C. Martins and M.P.D. Monteiro Marques (eds.), Kluwer Academic Publishers, Dordrecht 2002, 333-346.

[25] M. Raous, M. Schryve and M. Cocou, Recoverable adhesion and friction. Proceedings of the Second International Conference on Nonsmooth/Nonconvex Mechanics with Applications in Engineering, Thessaloniki, Greece, July 7-8, 2006, C.C. Baniotopoulos (ed.), Ziti Publisher, Greece 2006, 165-172.

[26] M. Schryve, Modèle d'adhésion cicatrisante et applications au contact verre/élastomère. PhD Thesis, Université de Provence, 2008.

[27] J. Simon, Compact sets in the space $L^{p}(0, T ; B)$. Ann. Mat. Pura Applic. 146 (1987), 65-96.

[28] M. Sofonea, W. Han and M. Shillor, Analysis and Approximation of Contact Problems with Adhesion or Damage. Chapman\&Hall/CRC, Boca Raton, 2006. 